\title{
Role of ground-state correlations in hypernuclear nonmesonic weak decay
}

\author{
E. Bauer ${ }^{1,2, *}$ and G. Garbarino ${ }^{3}$ \\ ${ }^{1}$ Departamento de Física, Universidad Nacional de La Plata, C. C. 67, 1900 La Plata, Argentina \\ ${ }^{2}$ Instituto de Física La Plata, CONICET, 1900 La Plata, Argentina \\ ${ }^{3}$ Dipartimento di Fisica Teorica, Università di Torino, and INFN, Sezione di Torino, I-10125 Torino, Italy
}

(Received 23 July 2009; revised manuscript received 30 March 2010; published 18 June 2010)

\begin{abstract}
The contribution of ground-state correlations (GSCs) to the nonmesonic weak decay of ${ }_{\Lambda}^{12} \mathrm{C}$ and other medium to heavy hypernuclei is studied within a nuclear-matter formalism implemented in a local-density approximation. We adopt a weak transition potential including the exchange of the complete octets of pseudoscalar and vector mesons, as well as a residual strong interaction modeled on the Bonn potential. Leading GSC contributions, at first order in the residual strong interaction, are introduced on the same footing for all isospin channels of oneand two-nucleon induced decays. Together with fermion antisymmetrization, GSCs turn out to be important for an accurate determination of the decay widths. Besides opening the two-nucleon stimulated decay channels, for ${ }_{\Lambda}^{12} \mathrm{C}$ GSCs are responsible for $14 \%$ of the rate $\Gamma_{1}$ while increasing the $\Gamma_{n} / \Gamma_{p}$ ratio by $4 \%$. Our final results for ${ }_{\Lambda}^{12} \mathrm{C}$ are $\Gamma_{\mathrm{NM}}=0.98, \Gamma_{n} / \Gamma_{p}=0.34$, and $\Gamma_{2} / \Gamma_{\mathrm{NM}}=0.26$. The saturation property of $\Gamma_{\mathrm{NM}}$ with increasing hypernuclear mass number is clearly observed. The agreement with data of our predictions for $\Gamma_{\mathrm{NM}}, \Gamma_{n} / \Gamma_{p}$, and $\Gamma_{2}$ is rather good.
\end{abstract}

DOI: 10.1103/PhysRevC.81.064315

\section{INTRODUCTION}

Nuclear systems with strangeness play an important role in modern nuclear and hadronic physics [1]. They imply important links with astrophysical processes and observables, as well as with QCD, the underlying theory of strong interactions. Various strange nuclear systems can be studied in the laboratory, ranging from hypernuclei and kaonic nuclei to exotic hadronic states such as strangelets, $H$-dibaryons, and pentaquark baryons. Strangeness production can also be investigated in relativistic heavy-ion collision experiments, whose main aim is to establish the existence of a quark-gluon plasma. Moreover, the cold and dense matter contained in neutron stars is expected to be composed of strange hadrons, in the form of hyperons and Bose-Einstein condensates of kaons, and eventually by strange quark matter for sufficiently dense systems.

The existence of hypernuclei-bound systems of nonstrange and strange baryons-opens up the possibility to study the hyperon-nucleon and hyperon-hyperon interactions in both the strong and the weak sectors. In turn, such interactions are important inputs, for instance, when investigating the macroscopic properties (masses and radii) of neutron stars. The best studied hypernuclei contain a single $\Lambda$ hyperon. In a nucleus the $\Lambda$ can decay by emitting a nucleon and a pion (mesonic mode) as it happens in free space, but its (weak) interaction with the nucleons opens new channels which are indicated as nonmesonic decay modes (for recent reviews, see Refs. [2-6]). These are the dominant decay channels of medium-heavy hypernuclei, where the mesonic decay is disfavored by the Pauli blocking effect on the outgoing nucleon. In particular, one can distinguish between one- and two-body induced decays, $\Lambda N \rightarrow n N$ and $\Lambda N N \rightarrow n N N$. The hypernuclear lifetime is given in terms of the mesonic $\left(\Gamma_{M}=\Gamma_{\pi^{-}}+\Gamma_{\pi^{0}}\right)$

*bauer@ fisica.unlp.edu.ar
PACS number(s): 21.80.+a, 25.80.Pw, 12.15.-y

and nonmesonic decay widths $\left(\Gamma_{\mathrm{NM}}=\Gamma_{1}+\Gamma_{2}\right)$ by $\tau=$ $\hbar / \Gamma_{\mathrm{T}}=\hbar /\left(\Gamma_{\mathrm{M}}+\Gamma_{\mathrm{NM}}\right)$. The various isospin channels contribute to the one- and two-nucleon induced nonmesonic rates as follows: $\Gamma_{1}=\Gamma_{n}+\Gamma_{p} \equiv \Gamma(\Lambda n \rightarrow n n)+\Gamma(\Lambda p \rightarrow n p)$ and $\Gamma_{2}=\Gamma_{n n}+\Gamma_{n p}+\Gamma_{p p} \equiv \Gamma(\Lambda n n \rightarrow n n n)+\Gamma(\Lambda n p \rightarrow$ $n n p)+\Gamma(\Lambda p p \rightarrow n p p)$.

One should note that, strictly speaking, the only observables in hypernuclear weak decay are the lifetime $\tau$, the mesonic rates $\Gamma_{\pi^{-}}$and $\Gamma_{\pi^{0}}$ (hence the total nonmesonic rate $\Gamma_{\mathrm{NM}}$ ), and the spectra of the emitted particles (nucleons, pions, and photons). None of the aforementioned nonmesonic partial decay rates $\left(\Gamma_{n}, \Gamma_{p}, \Gamma_{n p}\right.$, etc. $)$ is an observable from a quantum-mechanical point of view. The reason is as follows. Each one of the possible elementary nonmesonic decays occurs in the nuclear environment; thus, subsequent finalstate interactions (FSIs) modify the quantum numbers of the weak decay nucleons and new, secondary nucleons are emitted as well. This prevents the measurement of any of the nonmesonic partial decay rates. Instead, the total width $\Gamma_{\mathrm{T}}$ can be measured; being an inclusive quantity, for its measurement one has to detect any of the possible products of either mesonic or nonmesonic decays (typically protons from nonmesonic decays) as a function of time and then fit the observed distribution with an exponential decay law. The fact that the detected particles undergo FSI does not appreciably alter the lifetime measurement, because strong interactions proceed on a much shorter time scale than weak decays and $\tau^{\text {measured }}=\tau+\tau^{\text {strong }} \simeq \tau \equiv \hbar / \Gamma_{\mathrm{T}}$.

To achieve a proper knowledge of the various decay mechanisms (in particular of the strangeness-changing baryonbaryon interactions), a meaningful comparison between theory and experiment must be possible. The preceding discussion shows that such a comparison requires the introduction of nonstandard definitions for the nonmesonic partial decay rates (which, as mentioned, are not quantum-mechanical observables). The only constraint that these definitions must meet is to be in accord with the experimental methods adopted for 
determining these rates. In our opinion, this point has not been adequately addressed in previous works and, among others, it has impacted on the well-known puzzle of the ratio $\Gamma_{n} / \Gamma_{p}$ between the neutron- and the proton-induced nonmesonic rates; the same applies to today's discussion of $\Gamma_{2} / \Gamma_{\mathrm{NM}}$.

To explain how the total nonmesonic rate can be measured in an experiment, we have to discuss first the measurement of the mesonic rates. The pion and nucleon emitted in a mesonic decay both have a momentum of about $100 \mathrm{MeV} / c$. Nucleons of a few MeV kinetic energy cannot be observed because they are below the experimental detection thresholds. Mesonic decays are thus identified by measuring pions $\left(\pi^{-}\right.$'s or $\pi^{0} \rightarrow$ $\gamma \gamma$ decays $)$. The mesonic width $\Gamma_{\pi^{-}}\left(\Gamma_{\pi^{0}}\right)$ is determined from the observed $\pi^{-}\left(\pi^{0} \rightarrow \gamma \gamma\right)$ energy spectra and the total width $\Gamma_{\mathrm{T}}$. For instance, one obtains $\Gamma_{\pi^{-}}^{\exp }=\left(N_{\pi^{-}} / N_{\mathrm{hyp}}\right) \Gamma_{\mathrm{T}}^{\exp }$, $N_{\pi^{-}}$being the total number of detected $\pi^{-}$'s and $N_{\text {hyp }}$ the total number of produced hypernuclei (both these numbers are corrected for the detection efficiencies and the detector acceptances implied in the measurements). The mesonic rates measured in this way thus include the effect of in-medium pion renormalization. Theoretical models [7,8] also taking into account distorted pion waves obtained mesonic widths in agreement with the experimental values (in particular, the importance of the pion wave-function distortion was first demonstrated in the works of Ref. [7]).

The experimental total nonmesonic rate is then obtained as the difference between the total and the mesonic rates, $\Gamma_{\mathrm{NM}}^{\exp }=$ $\Gamma_{\mathrm{T}}^{\exp }-\Gamma_{\mathrm{M}}^{\exp }$. The determination of the so-called experimental value of $\Gamma_{n} / \Gamma_{p}$ is much more involved. Indeed, because it is not an observable, this ratio must be extracted from the nucleon emission spectra; this requires some theoretical input $[9,10]$. FSIs are very important for the nonmesonic processes and nucleons that have or have not suffered FSIs are indistinguishable between each other. A theoretical simulation of nucleon FSI is thus needed and, in principle, a coherent sum of both kinds of nucleons must be considered when evaluating the spectra. Generally, FSIs are accounted for by an intranuclear cascade (INC) model [11], which is a semiclassical scheme representing the effect of nucleon rescattering. To avoid double counting, the nucleon correlations implied in the rescattering must not be included in the evaluation of the decay widths. This important question has never been discussed in the literature. Although it is not the aim of the present contribution to progress on this issue, we analyze its main features in the next section.

The relevance of the nucleon-nucleon interaction in the nonmesonic decay is twofold: It concerns both the nucleon rescattering process which allows one to extract the experimental values for the decay widths, and it also affects the evaluation of the decay widths themselves. As an example of the last point, the decay width $\Gamma_{2}$ results from nuclear correlations. The inclusion of these correlations in the evaluation of the decay widths is a difficult task in a microscopic model. The calculation of $\Gamma_{2}$ was addressed in an approximate way in the pioneering work of Alberico et al. [12], where a phenomenological description of the two-particle, two-hole $(2 \mathrm{p} 2 \mathrm{~h})$ polarization propagator was introduced by adapting previous results by the same authors on electron scattering off nuclei to nuclear pion absorption. Thereafter, in Refs. $[13,14]$, an approximate phase-space argument for the
$2 \mathrm{p} 2 \mathrm{~h}$ configurations and data on real pion absorption in nuclei were adopted for an improved evaluation of $\Gamma_{2}$. The first microscopic calculation of the nonmesonic rates was attempted in Ref. [15] by means of a path integral method which allowed a classification of the $2 \mathrm{p} 2 \mathrm{~h}$ contributions according to the so-called boson loop expansion. For technical reasons, it was not possible to separate the total width $\Gamma_{\mathrm{NM}}=\Gamma_{1}+\Gamma_{2}$ into the partial contributions $\Gamma_{1}$ and $\Gamma_{2}$ in that work.

In the microscopic evaluation of $\Gamma_{1}$ and $\Gamma_{2}$ it is possible to differentiate between correlations acting on the initial or ground state [resulting in the so-called ground-state correlations (GSCs)] and correlations on the final state (they are thus part of FSIs). To the best of our knowledge, there is no microscopic calculation of the FSI contribution to the decay widths. As already mentioned, for the inclusion of FSIs in the evaluation of the decay widths we have the additional problem of a potential double counting with the rescattering process. At variance, the GSCs are less controversial because they only contribute to the decay widths. The effect of GSCs, which has been studied in some former works [16-19], is the main subject of the present work. Up to now, GSCs have been discussed only in connection with the two-nucleon stimulated decay. In the present work we estimate for the first time their effect on the one-nucleon induced decay.

More specifically, we study the nonmesonic weak decay of hypernuclei ranging from ${ }_{\Lambda}^{11} \mathrm{~B}$ to ${ }_{\Lambda}^{208} \mathrm{~Pb}$ by using a nuclear-matter approach implemented in a local-density approximation. All the possible isospin channels for one- and two-body induced mechanisms are included in a microscopic approach based on the evaluation of Goldstone diagrams. The partial decay rates are derived by starting from a two-body weak transition potential. Leading-order GSC contributions are introduced on the same ground for one- and two-nucleon induced processes. The weak transition potential contains the exchange of mesons of the pseudoscalar and vector octets, $\pi, \eta, K, \rho, \omega$, and $K^{*}$, while for the nucleon-nucleon strong interaction contributing to GSCs we adopt a Bonn potential with the exchange of $\pi, \rho, \sigma$, and $\omega$ mesons. The general formalism we use was established in Refs. [16-20].

The article is organized as follows. In Sec. II we start with general considerations about FSIs, the definitions we employ for the weak decay rates, as well as the method usually adopted for the determination of the partial rates (for instance $\Gamma_{n} / \Gamma_{p}$ ) from data on nucleon spectra. In Sec. III we introduce the general framework for the evaluation of the one- and two-nucleon induced decay widths with the inclusion of GSC. Explicit expressions for the considered GSC diagrams contributing to the one-nucleon induced rates are given in Sec. IV and Appendix A. Then, in Sec. V our results are presented and compared with data. Finally, in Sec. VI some conclusions are given.

\section{CONSIDERATIONS ON FSI EFFECTS AND THE DETERMINATION OF THE WEAK DECAY RATES}

The $\Gamma_{n} / \Gamma_{p}$ ratio is defined as the ratio between the total number of primary (i.e., weak decay) neutron-neutron and neutron-proton pairs, $N_{n n}^{\mathrm{wd}}$ and $N_{n p}^{\mathrm{wd}}$, emerging from the processes $\Lambda n \rightarrow n n$ and $\Lambda p \rightarrow n p$, respectively. Owing to nucleon FSIs and two-body induced decays, the following 
inequality is expected between $\Gamma_{n} / \Gamma_{p}$ and the observables $n n$ and $n p$ coincidence numbers, $N_{n n}$ and $N_{n p}[9]^{1}$ :

$$
\frac{\Gamma_{n}}{\Gamma_{p}} \equiv \frac{N_{n n}^{\mathrm{wd}}}{N_{n p}^{\mathrm{wd}}} \neq \frac{N_{n n}}{N_{n p}} .
$$

$N_{n n} / N_{n p}$ is surely a quantum-mechanical observable: Generally, its measurement is affected by thresholds on the nucleon energies and the pair opening angles [6,21,22]. The preceding inequality and the discussion of Ref. [9] clearly establish the nonobservable character of $\Gamma_{n} / \Gamma_{p}$. Theoretical models are thus required to determine the "experimental" value of $\Gamma_{n} / \Gamma_{p}$ from a measurement of $N_{n n} / N_{n p}$. This unusual procedure for determining $\left(\Gamma_{n} / \Gamma_{p}\right)^{\exp }$ makes complete sense provided different models are at disposal and lead to the same extracted ratio: Only in such a case one is allowed to define this value as the experimental result for $\Gamma_{n} / \Gamma_{p}$. In the present section we go deeper into questions of this kind to show some ambiguities that need to be emphasized for a meaningful comparison between theory and experiment.

Each one of the nonmesonic weak decay channels takes place by the emission of two or three primary nucleons. These nucleons then propagate within the nuclear environment and cannot be measured. The strong interactions with the surrounding nucleons can change the charge and the energymomentum of the primary nucleons; some of them can be absorbed by the medium and the emission of additional (secondary) nucleons can occur as well. All these processes are generically designated as FSIs: the observable nucleon spectra, that is, $N_{n n}$ and $N_{n p}$ in Eq. (1), are crucially affected by them.

FSIs pertain to the same quantum-mechanical problem which starts with the weak interaction involving the $\Lambda$ hyperon and ends with the detection of the particles emitted by the hypernucleus. In a strict quantum-mechanical scheme, FSIs cannot thus be disentangled from the weak interaction part of the problem: This is an analogous way of expressing the fact that the partial weak decay rates are not measurable. As explained in the Introduction, only the total nonmesonic rate is an observable.

The question thus arises of which FSI contributions have to be included in the calculation of the decay rates. A first kind of FSI that one has to consider consist of the baryon-baryon short-range correlations (SRCs) acting on the nucleon-nucleon final state. SRCs for both the initial and the final states (as well as mean field effects on the singleparticle wave functions) are genuine contributions to the decay rates. This is so because SRCs act on two-particle states involving primary baryons and therefore do not alter the number nor the species of nucleons involved in the weak decays. Given the nonobservable character of the partial decay rates, one has no prescription to determine which kind of FSI has to be included in the calculations of these rates. Although we make here some considerations about this question, which

\footnotetext{
${ }^{1}$ Note that an analogous inequality exists between $\Gamma_{n} / \Gamma_{p}$ and the ratio between the total number of emitted neutrons and protons, $N_{n} / N_{p}$ [10]. For the present discussion, either of these two expressions is suitable.
}

does not have a simple solution, we believe that a complete answer to it goes beyond the present contribution.

To analyze this important point in more detail, it is convenient to consider the way FSI contributions are included in the calculation of observables such as the nucleon spectra. To date, nucleon rescattering effects have always been taken into account in an approximate way, by resorting to INC models [11] rather than by solving the exact quantum-mechanical problem (as far as we know, the only attempts to develop a microscopic many-body model for FSIs are the ones of Refs. [23-25]). The INC approach is based on a semiclassical picture in which both the nucleon de Broglie wavelength $\lambda=h / p$ and the range of the nucleon-nucleon hard-core interaction $r_{N N}$ are much smaller than the in-medium nucleon mean free path. Subsequent nucleon-nucleon collisions in the rescattering process are thus independent of one another. Between collisions the nucleons are treated as classical particles: They are on shell, with definite positions and momenta, and move along straight-line trajectories under the influence of a local mean field. Each collision is described by free-space nucleon-nucleon cross sections $\sigma_{N N}$ corrected to account for medium effects such as Pauli blocking and nucleon Fermi motion. Roughly speaking, collisions occur whenever two nucleons come closer than $l \simeq 2 r_{N N} \simeq \sqrt{\sigma_{N N} / \pi}$; the scattering angle is chosen randomly according to the freespace differential nucleon-nucleon cross sections. Long-range interactions (responsible for instance of the nuclear mean-field potential) are thus neglected in INC calculations; short-range nucleon-nucleon interactions (involved in collisions) play a dominant role instead.

This discussion makes evident two aspects of the INC rescattering that are important in our analysis of the relevance of FSIs for the microscopic calculation of the decay widths: (i) nucleons that propagate between two successive interaction points of the cascade are almost on shell; (ii) the nucleon collisions proceed by the (repulsive) short-range part of the nucleon-nucleon interaction.

Let us now analyze the same rescattering problem in terms of a microscopic quantum-mechanical calculation. In general terms, one could wonder if it is possible to identify those quantum-mechanical contributions whose classical limit leads to a factorization between the weak decay process and the INC rescattering. This is a relevant question because in the theoretical evaluation of the nonmesonic decay rates FSI contributions to the INC rescattering must not be included; one indeed aims to extract the contribution of the elementary $\Lambda N \rightarrow n N$ and $\Lambda n n \rightarrow n N N$ processes by studying hypernuclear decay. Unfortunately, this question does not seem to have a simple answer, at least from a technical standpoint. Consider indeed the $\Lambda$ self-energy diagram in Fig. 1(a) with the pole on the $3 \mathrm{p} 2 \mathrm{~h}$ configuration, as indicated by the dotted line. This is a (time-ordered) Goldstone diagram, where the weak transition potential $V^{\Lambda N \rightarrow N N}$, which is a two-body operator, produces an intermediate $2 \mathrm{p} 1 \mathrm{~h}$ configuration; afterward, the action of the nucleon-nucleon strong interaction $V^{N N}$ creates a further $1 \mathrm{p} 1 \mathrm{~h}$ pair and leads to a $3 \mathrm{p} 2 \mathrm{~h}$ final state. In terms of amplitudes, $V^{\Lambda N \rightarrow N N}$ produces two nucleons, one of which then strongly interacts with another nucleon, ending in the emission of three nucleons. Because the potential $V^{N N}$ acts after $V^{\Lambda N \rightarrow N N}$, 
(a)

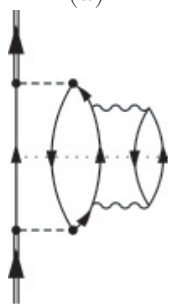

(b)



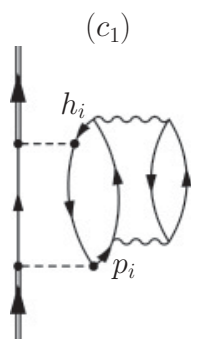

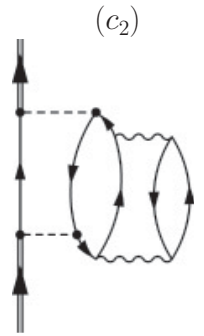

FIG. 1. Goldstone diagrams for three-nucleon emission originated by FSI (a), 2p2h GSC (b), and FSI-GSC interferences (c1) and (c2). The dashed and wavy lines stand for the potentials $V^{\Lambda N \rightarrow N N}$ and $V^{N N}$, respectively. Part (a) has poles on the $2 \mathrm{p} 1 \mathrm{~h}$ and $3 \mathrm{p} 2 \mathrm{~h}$ configurations, while part (b) has a single pole on the $3 \mathrm{p} 2 \mathrm{~h}$ configuration. For the discussion made in the text, only the $3 \mathrm{p} 2 \mathrm{~h}$ poles indicated by the dotted lines are relevant. The interference diagrams (c1) and (c2) are vanishing because $p_{i}=h_{i}$.

Fig. 1(a) contains by definition a FSI effect. Note that the idea of an interaction taking place after or before another one is a valid statement here as we are working with Goldstone diagrams. In our discussion we are using Goldstone diagrams instead of Feynman diagrams because our aim here is to isolate those nucleon FSI effects that contribute to nucleon spectra but not to the decay widths (in other words, the use of Goldstone diagrams is attributable to the nonobservability of the decay rates). According to the considerations made in the previous paragraph, Fig. 1(a) partially contributes to nucleon rescattering: This occurs when the nucleon connecting $V^{\Lambda N \rightarrow N N}$ with $V^{N N}$ (in both the upper and the lower parts of the diagram) is almost on shell and $V^{N N}$ contains only the repulsive part of the nucleon-nucleon potential (i.e., the exchange of heavy mesons). For all the other kinematics and dynamical conditions, Fig. 1(a) in principle contributes to the decay widths. This conclusion agrees with the method normally adopted to extract the partial decay widths (for instance, $\Gamma_{n} / \Gamma_{p}$ and $\left.\Gamma_{2} / \Gamma_{\mathrm{NM}}\right)$ from the measured nucleon spectra: The procedure of correcting these spectra for the nucleon rescattering supplies the distributions of primary nucleons from which one can easily deduce the partial decay rates.

We therefore see that each Goldstone diagram with at least one $V^{N N}$ acting after the $V^{\Lambda N \rightarrow N N}$ may partially contribute to the decay rates. It is important to stress that such contributions originate from the method adopted to determine these rates from the measured nucleon spectra. This method, which is obviously the only one available to us to define the decay widths, leads us to an unfortunate situation. On the one hand, the inclusion of diagrams like Fig. 1(a) in the calculation of the rates would introduce some unknown double counting with the INC rescattering and would probably lead to an overestimation of the two-nucleon induced decay rate $\Gamma_{2}$. On the other hand, by neglecting these diagrams, one may underestimate $\Gamma_{2}$. Given the difficulty in identifying accurately the parts of Goldstone diagrams contributing to the decay widths, as an operating procedure it seems more convenient to neglect in the present work all those diagrams [like Fig. 1(a)] embedding nucleon rescattering effects: The assumption that can at present justify this approximation being that these diagrams give a relevant contribution to the rescattering (this is demonstrated by the microscopic approach of [25]) and thus their effect on $\Gamma_{2}$ should be limited. Furthermore, the comparison of our results with the experimental values of $\Gamma_{2}$ could be used as a parameter for the assessment of the goodness of this approximation.

The problem posed by the impossibility of identifying diagrams that only contribute to the decay rates can be overcome if one instead evaluates the nucleon emission spectra, which are observable and thus must be evaluated in terms of Feynman diagrams. A microscopic model for the evaluation of these spectra was developed in Refs. [23-25]. In particular, the diagram in Fig. 1(a) (together with the other FSI diagrams at the same order in $V^{N N}$ ) was evaluated in Ref. [25], finding a rather important contribution to the single-nucleon spectra at low energy, that is, to nucleon rescattering.

In the present contribution our main concern is instead the role played by GSCs in the evaluation of the decay rates. As we show in the next two sections, the addition of GSCs is a rather complex task in a microscopic model. A typical GSC contribution to $\Gamma_{2}$ is depicted in Fig. 1(b). It corresponds to an amplitude in which the $\Lambda$ decays by interacting with a correlated nucleon pair. Because the nucleon-nucleon interaction takes place before the action of the weak transition, this diagram correspond to a GSC and is a genuine contribution to the decay rates. Note that the Goldstone diagrams in Figs. 1(a)1(c2) are different time orderings of the same Feynman diagram. Figures 1(a) and 1(b) have a positive contribution from the $3 \mathrm{p} 2 \mathrm{~h}$ cut, because each of them results from the square of a transition amplitude. At variance, Figs. 1(c1) and 1(c2) are both identically vanishing because the momenta carried by the particle $p_{i}$ and the hole $h_{i}$ indicated in the figure have the same value, that is, the conditions $p_{i}>k_{F}$ and $h_{i} \leqslant k_{F}$, where $k_{F}$ is the Fermi momentum, cannot be fulfilled simultaneously.

It should be emphasized that the comparison between our results for the decay widths and the corresponding experimental values makes complete sense. This comparison, addressed in Sec. V, tests the consistency in our model between the decay widths and the experimental values of the rates obtained by a deconvolution of the nucleon rescattering effects contained in the measured spectra.

\section{MANY-BODY TERMS IN THE NONMESONIC DECAY RATES}

Let us consider the one and two-body induced nonmesonic weak decay width for a $\Lambda$ hyperon with four-momentum $k=$ $\left(k_{0}, \mathbf{k}\right)$ inside infinite nuclear matter with Fermi momentum $k_{F}$. In a schematic way, one can write

$$
\Gamma_{1(2)}\left(k, k_{F}\right)=\sum_{f}\left|\left\langle f\left|V^{\Lambda N \rightarrow N N}\right| 0\right\rangle_{k_{F}}\right|^{2} \delta\left(E_{f}-E_{0}\right),
$$

where $|0\rangle_{k_{F}}$ and $|f\rangle$ are the initial hypernuclear ground state (whose energy is $E_{0}$ ) and the possible $2 \mathrm{p} 1 \mathrm{~h}$ or $3 \mathrm{p} 2 \mathrm{~h}$ final states, respectively. The $2 \mathrm{p} 1 \mathrm{~h}(3 \mathrm{p} 2 \mathrm{~h})$ final states define $\Gamma_{1}\left(\Gamma_{2}\right)$. The final-state energy is $E_{f}$ and $V^{\Lambda N \rightarrow N N}$ is the two-body weak transition potential.

The decay rates for a finite hypernucleus are obtained by the local-density approximation [26], that is, after averaging the preceding partial width over the $\Lambda$ momentum distribution in the considered hypernucleus, $\left|\widetilde{\psi}_{\Lambda}(\mathbf{k})\right|^{2}$, and over the local 
Fermi momentum, $k_{F}(r)=\left\{3 \pi^{2} \rho(r) / 2\right\}^{1 / 3}, \rho(r)$ being the density profile of the hypernuclear core. One thus has

$$
\Gamma_{1(2)}=\int d \mathbf{k}\left|\widetilde{\psi}_{\Lambda}(\mathbf{k})\right|^{2} \int d \mathbf{r}\left|\psi_{\Lambda}(\mathbf{r})\right|^{2} \Gamma_{1(2)}\left[\mathbf{k}, k_{F}(r)\right],
$$

where for $\psi_{\Lambda}(\mathbf{r})$, the Fourier transform of $\widetilde{\psi}_{\Lambda}(\mathbf{k})$, we adopt the $1 s_{1 / 2}$ harmonic oscillator wave function with frequency $\hbar \omega\left(=10.8 \mathrm{MeV}\right.$ for $\left.{ }_{\Lambda}^{12} \mathrm{C}\right)$ adjusted to the experimental energy separation between the $s$ and $p \Lambda$ levels in the considered hypernucleus. The $\Lambda$ total energy in Eqs. (2) and (3) is given by $k_{0}=m_{\Lambda}+\mathbf{k}^{2} /\left(2 m_{\Lambda}\right)+V_{\Lambda}, V_{\Lambda}\left(=-10.8 \mathrm{MeV}\right.$ for $\left.{ }_{\Lambda}^{12} \mathrm{C}\right)$ being a binding energy term.

Because $V^{\Lambda N \rightarrow N N}$ is a two-body operator, the emission of two nucleons is originated either from the Hartree-Fock vacuum or from GSCs induced by the nucleon-nucleon interaction. At variance, the emission of three nucleons can be achieved only when $V^{\Lambda N \rightarrow N N}$ acts over a GSC. It is therefore convenient to introduce the following hypernuclear ground-state wave function [17]:

$$
\begin{aligned}
|0\rangle_{k_{F}}= & \mathcal{N}\left(k_{F}\right)\left(|\rangle-\sum_{p, h, p^{\prime}, h^{\prime}} \frac{\left\langle p h p^{\prime} h^{\prime}\left|V^{N N}\right|\right\rangle_{D+E}}{\varepsilon_{p}-\varepsilon_{h}+\varepsilon_{p^{\prime}}-\varepsilon_{h^{\prime}}}\left|p h p^{\prime} h^{\prime}\right\rangle\right) \\
& \times \otimes\left|p_{\Lambda}\right\rangle,
\end{aligned}
$$

where |\rangle is the uncorrelated core ground-state wave function, that is, the Hartree-Fock vacuum, and the second term in the right-hand side represents $2 \mathrm{p} 2 \mathrm{~h}$ correlations and contains both direct $(D)$ and exchange $(E)$ matrix elements of the nuclear residual interaction $V^{N N}$. Besides, $\left|p_{\Lambda}\right\rangle$ is the normalized state of the $\Lambda$, the particle and hole energies are denoted by $\varepsilon_{i}$ and

$$
\mathcal{N}\left(k_{F}\right)=\left(1+\sum_{p, h, p^{\prime}, h^{\prime}}\left|\frac{\left\langle p h p^{\prime} h^{\prime}\left|V^{N N}\right|\right\rangle_{D+E}}{\varepsilon_{p}-\varepsilon_{h}+\varepsilon_{p^{\prime}}-\varepsilon_{h^{\prime}}}\right|^{2}\right)^{-1 / 2}
$$

is the ground-state normalization function. The particular labeling of Eqs. (4) and (5) is explained in Fig. 2. The explicit expression for $\mathcal{N}\left(k_{F}\right)$ is given in Ref. [18].

By inserting Eq. (4) into Eq. (2), for $\Gamma_{1}$ one obtains

$$
\begin{aligned}
\Gamma_{1}\left(\mathbf{k}, k_{F}\right)= & \mathcal{N}^{2}\left(k_{F}\right) \sum_{f} \delta\left(E_{f}-E_{0}\right) \mid\left\langle f\left|V^{\Lambda N \rightarrow N N}\right| p_{\Lambda}\right\rangle_{D+E} \\
& -\sum_{p, h, p^{\prime}, h^{\prime}}\left\langle f\left|V^{\Lambda N \rightarrow N N}\right| p h p^{\prime} h^{\prime} ; p_{\Lambda}\right\rangle_{D+E} \\
& \times\left.\frac{\left\langle p h p^{\prime} h^{\prime} ; p_{\Lambda}\left|V^{N N}\right| p_{\Lambda}\right\rangle_{D+E}}{\varepsilon_{p}-\varepsilon_{h}+\varepsilon_{p^{\prime}}-\varepsilon_{h^{\prime}}}\right|^{2},
\end{aligned}
$$

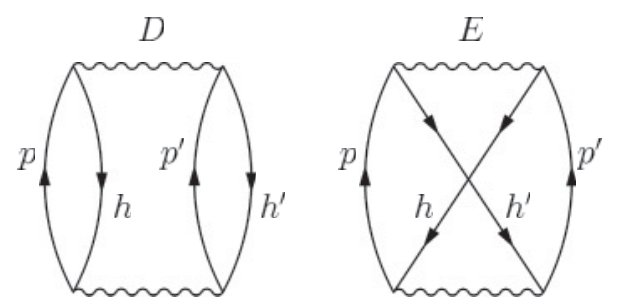

FIG. 2. Direct $(D)$ and exchange $(E)$ Goldstone diagrams for the 2p2h GSC induced by the nuclear residual interaction $V^{N N}$. the final states $|f\rangle$ being restricted to 2 p1h states. For $\Gamma_{2}$ one has

$$
\begin{aligned}
\Gamma_{2}\left(\mathbf{k}, k_{F}\right)= & \mathcal{N}^{2}\left(k_{F}\right) \sum_{f} \delta\left(E_{f}-E_{0}\right) \\
& \times \mid \sum_{p, h, p^{\prime}, h^{\prime}}\left\langle f\left|V^{\Lambda N \rightarrow N N}\right| p h p^{\prime} h^{\prime} ; p_{\Lambda}\right\rangle_{D+E} \\
& \times\left.\frac{\left\langle p h p^{\prime} h^{\prime} ; p_{\Lambda}\left|V^{N N}\right| p_{\Lambda}\right\rangle_{D+E}}{\varepsilon_{p}-\varepsilon_{h}+\varepsilon_{p^{\prime}}-\varepsilon_{h^{\prime}}}\right|^{2}
\end{aligned}
$$

where the final states are given by $3 \mathrm{p} 2 \mathrm{~h}$ states. Note that all the matrix elements of $V^{N N}$ and $V^{\Lambda N \rightarrow N N}$ appear in the antisymmetrized form.

Let us focus now on the kind of diagrams contributing to $\Gamma_{1}$ and $\Gamma_{2}$. This discussion is done in terms of transition amplitudes rather than self-energies. In Fig. 3 we report some of the most representative transition amplitudes that contribute to $\Gamma_{1}$. All diagrams but Fig. 3(a) are originated by a GSC. Only the contribution of Fig. 3(a) to $\Gamma_{1}$ has been calculated microscopically up to now. The Figs. 3(b1)-3(b3) represent typical $2 \mathrm{p} 2 \mathrm{~h}$ correlations. Figure 3(c) is a contact term involving a $\pi \pi N N$ strong vertex, while Figs. 3(d) and $3(\mathrm{~d} 2)$ represent the contribution of the $\Delta(1232)$ resonance. It should be mentioned that there has been a great deal of controversy around the theoretical determination of the
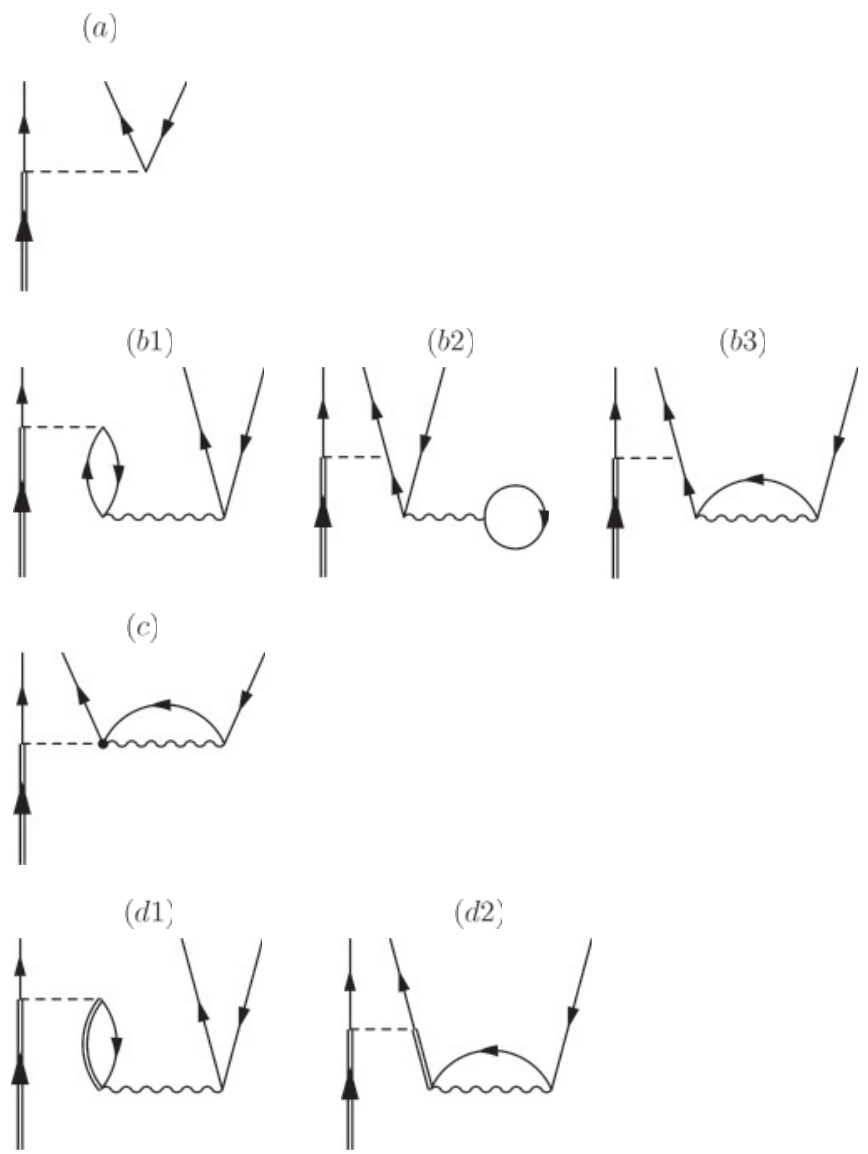

FIG. 3. Transition amplitudes contributing to $\Gamma_{1}$. A double line (without arrow) represents the $\Delta(1232)$ resonance. 


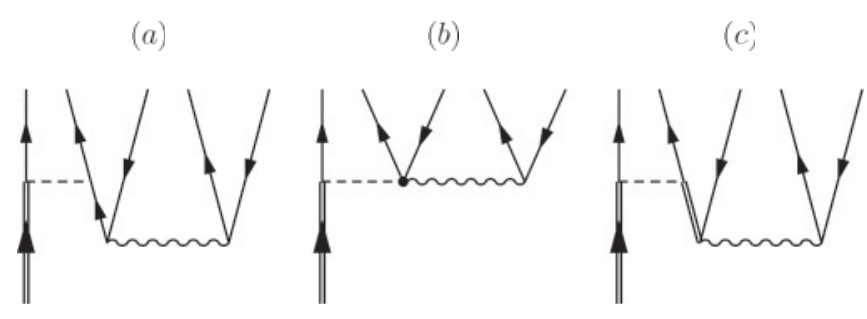

FIG. 4. Transition amplitudes contributing to $\Gamma_{2}$.

$\Gamma_{n} / \Gamma_{p}$ ratio and the challenging comparison with data. In these discussions, all theoretical efforts were devoted to the Fig. 3(a) term only; the remaining ones have simply been ignored.

A similar analysis can be done for $\Gamma_{2}$ starting from the amplitudes of Fig. 4. Again, only the term in Fig. 4(a) has been evaluated up to now in microscopic calculations [16]. Note that the diagrams in Figs. 3 and 4 are only representative cases. For instance, the amplitude of Fig. 5 should also be included when calculating $\Gamma_{1}$. Unlike the other amplitudes of Figs. 3 and 4, the one in Fig. 5 involves a strong interaction $V^{\Lambda N}$ between the $\Lambda$ and a $1 \mathrm{p} 1 \mathrm{~h}$ pair (i.e., a $1 \mathrm{p} 1 \mathrm{~h}$ GSC) and then the usual action of the weak transition potential. Rather than calculating this diagram explicitly, it is common to include it in an effective way by incorporating short-range correlation effects in the weak potential $V^{\Lambda N \rightarrow N N}$; we follow this practice. Other amplitudes are relevant in a microscopic approach. In Fig. 4(a) the weak transition potential can also be connected to a hole line [16]. In addition, because $V^{N N}$ and $V^{\Lambda N \rightarrow N N}$ are two-body operators whose matrix elements are antisymmetrized, Pauli exchange terms must be considered as well [18].

All the diagrams in Figs. 3 and 4 have the same initial state, which is the hypernuclear ground state. The final state of the diagram in Fig. 3 (Fig. 4) is a 2 p1h ( $3 p 2 h)$ state. To obtain the various decay widths, all diagrams representing transition amplitudes with the same initial and final states are added and then squared. For instance, from Fig. 4 one obtains a total of six direct diagrams: the square of each individual amplitude plus the three interference terms. For the amplitudes in Fig. 3 there is a total of 28 different direct terms. In addition, antisymmetrization considerably increases the number of diagrams. Our previous work indicates that a complete microscopic evaluation is important for several reasons. First, a raw estimation of a remarkable amount of different diagrams makes the final result quite uncertain. Second, there is no

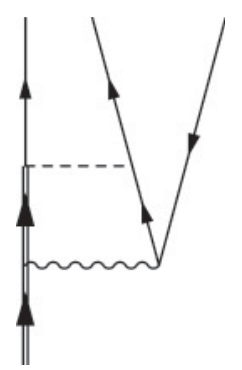

FIG. 5. Transition amplitude contributing to $\Gamma_{1}$ and involving a strong interaction $V^{\Lambda N}$ between the hyperon and a $1 \mathrm{p} 1 \mathrm{~h}$ pair. ground to evaluate differently the diagrams originated from Fig. 3 and those from Fig. 4: Once a microscopic calculation is performed for the square of diagrams (a) of Figs. 3 and 4, the same should be done for the remaining contributions, which are all leading-order GSC contributions.

In the present work, as a further step toward the calculation of the whole set of diagrams relevant for the nonmesonic decay, the one-nucleon induced widths originated from the sum of the transition amplitudes (a) plus (b1) of Fig. 3 are evaluated for the first time. Accordingly, the two-nucleon induced rates are instead obtained from the amplitude (a) of Fig. 4 by following Ref. [18]. Antisymmetrization is coherently applied to all contributions. In the next section we proceed with the formal derivation of the decay widths.

\section{FORMAL DERIVATION OF THE DECAY RATES $\Gamma_{n}$ AND $\Gamma_{p}$ INCLUDING GSC}

In Fig. 3 we have shown a set of amplitudes that contribute to the decay rate $\Gamma_{1}$ of Eq. (2). Only amplitude (a) has been evaluated explicitly up to now. In the present work we extend the microscopic approach to include amplitude (b1), which originates from GSC contributions that we expect to be important.

Before proceeding with the derivation of decay widths, it is convenient to give the expressions for the potentials. The weak transition potential $V^{\Lambda N \rightarrow N N}$ and the nuclear residual interaction $V^{N N}$ read

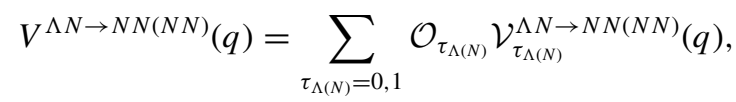

where the isospin dependence is given by

$$
\mathcal{O}_{\tau_{\Lambda(N)}}=\left\{\begin{array}{lll}
1 & \text { for } & \tau_{\Lambda(N)}=0 \\
\tau_{1} \cdot \tau_{2} & \text { for } & \tau_{\Lambda(N)}=1
\end{array}\right.
$$

The values 0 and 1 for $\tau_{\Lambda(N)}$ refer to the isoscalar and isovector parts of the interactions, respectively. The spin and momentum dependence of the weak transition potential is given by

$$
\begin{aligned}
\mathcal{V}_{\tau_{\Lambda}}^{\Lambda N} & \rightarrow N N(q) \\
= & \left(G_{F} m_{\pi}^{2}\right)\left\{S_{\tau_{\Lambda}}(q) \sigma_{1} \cdot \hat{\mathbf{q}}+S_{\tau_{\Lambda}}^{\prime}(q) \sigma_{2} \cdot \hat{\mathbf{q}}+P_{C, \tau_{\Lambda}}(q)\right. \\
& +P_{L, \tau_{\Lambda}}(q) \sigma_{1} \cdot \hat{\mathbf{q}} \sigma_{2} \cdot \hat{\mathbf{q}}+P_{T, \tau_{\Lambda}}(q)\left(\sigma_{1} \times \hat{\mathbf{q}}\right) \cdot\left(\sigma_{2} \times \hat{\mathbf{q}}\right) \\
& \left.+i S_{V, \tau_{\Lambda}}(q)\left(\sigma_{1} \times \sigma_{2}\right) \cdot \hat{\mathbf{q}}\right\},
\end{aligned}
$$

where the functions $S_{\tau_{\Lambda}}(q), S_{\tau_{\Lambda}}^{\prime}(q), P_{C, \tau_{\Lambda}}(q), P_{L, \tau_{\Lambda}}(q)$, $P_{T, \tau_{\Lambda}}(q)$, and $S_{V, \tau_{\Lambda}}(q)$, which include short-range correlations, can be adjusted to reproduce any weak transition potential. The corresponding expression for the nuclear residual interaction is given by

$$
\begin{aligned}
\mathcal{V}_{\tau_{N}}^{N N}(q)= & \frac{f_{\pi}^{2}}{m_{\pi}^{2}}\left\{\mathcal{V}_{C, \tau_{N}}(q)+\mathcal{V}_{L, \tau_{N}}(q) \sigma_{1} \cdot \hat{\mathbf{q}} \sigma_{2} \cdot \hat{\mathbf{q}}\right. \\
& \left.+\mathcal{V}_{T, \tau_{N}}(q)\left(\sigma_{1} \times \hat{\mathbf{q}}\right) \cdot\left(\sigma_{2} \times \hat{\mathbf{q}}\right)\right\},
\end{aligned}
$$

where the functions $\mathcal{V}_{C, \tau_{N}}(q), \mathcal{V}_{L, \tau_{N}}(q)$, and $\mathcal{V}_{T, \tau_{N}}(q)$ can also be adjusted to reproduce any nuclear residual interaction. In particular, $V^{\Lambda N \rightarrow N N}$ is represented by the exchange of the $\pi$, $\eta, K, \rho, \omega$, and $K^{*}$ mesons, within the formulation of Ref. [27], 
with strong coupling constants and cutoff parameters deduced from the Nijmegen soft-core interaction NSC97f of [28]. For $V^{N N}$ we have used a Bonn potential [29] in the framework of the parametrization presented in Ref. [30], which contains the exchange of $\pi, \rho, \sigma$, and $\omega$ mesons.

We give now explicit expressions for the partial decay width $\Gamma_{1}\left(\mathbf{k}, k_{F}\right)$ of Eq. (6), which for convenience is expressed in terms of its isospin components $\Gamma_{n}\left(\mathbf{k}, k_{F}\right)$ and $\Gamma_{p}\left(\mathbf{k}, k_{F}\right)$. Let us first rewrite Eq. (6) as follows:

$$
\Gamma_{n(p)}\left(\mathbf{k}, k_{F}\right)=\Gamma_{n(p)}^{0}\left(\mathbf{k}, k_{F}\right)+\Gamma_{n(p)}^{0-\mathrm{GSC}}\left(\mathbf{k}, k_{F}\right)+\Gamma_{n(p)}^{\mathrm{GSC}}\left(\mathbf{k}, k_{F}\right),
$$

where

$$
\begin{aligned}
\Gamma_{n(p)}^{0}\left(\mathbf{k}, k_{F}\right)= & \mathcal{N}^{2}\left(k_{F}\right) \sum_{f} \delta\left(E_{f}-E_{0}\right) \\
& \times\left|\left\langle f\left|V^{\Lambda N \rightarrow N N}\right| p_{\Lambda}\right\rangle_{D+E}\right|^{2}, \\
\Gamma_{n(p)}^{0-\mathrm{GSC}}\left(\mathbf{k}, k_{F}\right)= & -2 \mathcal{N}^{2}\left(k_{F}\right) \sum_{f} \sum_{p, h, p^{\prime}, h^{\prime}} \delta\left(E_{f}-E_{0}\right) \\
& \times\left\langle p_{\Lambda}\left|\left(V^{\Lambda N \rightarrow N N}\right)^{\dagger}\right| f\right\rangle_{D+E} \\
& \times\left\langle f\left|V^{\Lambda N \rightarrow N N}\right| p h p^{\prime} h^{\prime} ; p_{\Lambda}\right\rangle_{D+E} \\
& \times \frac{\left\langle p h p^{\prime} h^{\prime} ; p_{\Lambda}\left|V^{N N}\right| p_{\Lambda}\right\rangle_{D+E}}{\varepsilon_{p}-\varepsilon_{h}+\varepsilon_{p^{\prime}}-\varepsilon_{h^{\prime}}} \\
\Gamma_{n(p)}^{\mathrm{GSC}}\left(\mathbf{k}, k_{F}\right)= & \mathcal{N}^{2}\left(k_{F}\right) \sum_{f} \sum_{p, h, p^{\prime}, h^{\prime}} \delta\left(E_{f}-E_{0}\right) \\
& \times \mid\left\langle f\left|V^{\Lambda N \rightarrow N N}\right| p h p^{\prime} h^{\prime} ; p_{\Lambda}\right\rangle_{D+E} \\
& \times \frac{\left.\left\langle p h p^{\prime} h^{\prime} ; p_{\Lambda}\left|V^{N N}\right| p_{\Lambda}\right\rangle_{D+E}\right|^{2}}{\varepsilon_{p}-\varepsilon_{h}+\varepsilon_{p^{\prime}}-\varepsilon_{h^{\prime}}} \mid
\end{aligned}
$$

The first component, $\Gamma_{n(p)}^{0}$, is the contribution from the uncorrelated hypernuclear ground state, the third one, $\Gamma_{n(p)}^{\mathrm{GSC}}$, results from ground-state correlations, while $\Gamma_{n(p)}^{0-\mathrm{GSC}}$ is the interference term between correlated and uncorrelated ground states.

It is now convenient to consider the following decomposition, dictated by the isospin quantum number:

$$
\begin{aligned}
\Gamma_{n(p)}^{0}\left(\mathbf{k}, k_{F}\right)= & \sum_{P, Q=D, E} \Gamma_{n(p)}^{P Q}\left(\mathbf{k}, k_{F}\right) \\
= & \sum_{P, Q=D, E} \sum_{\tau_{\Lambda^{\prime}}, \tau_{\Lambda}=0,1} \mathcal{T}_{\tau_{\Lambda^{\prime}} \tau_{\Lambda}, n(p)}^{P Q} \\
& \times \Gamma_{\tau_{\Lambda^{\prime}} \tau_{\Lambda}}^{P Q}\left(\mathbf{k}, k_{F}\right), \\
\Gamma_{n(p)}^{0-\mathrm{GSC}}\left(\mathbf{k}, k_{F}\right)= & \sum_{P, Q, Q^{\prime}=D, E} \Gamma_{n(p)}^{P Q Q^{\prime}}\left(\mathbf{k}, k_{F}\right) \\
= & \sum_{P, Q, Q^{\prime}=D, E} \sum_{\tau_{\Lambda^{\prime}}, \tau_{\Lambda}, \tau_{N}=0,1} \mathcal{T}_{\tau_{\Lambda^{\prime}} \tau_{\Lambda} \tau_{N}, n(p)}^{P \tau^{\prime}} \\
& \times \Gamma_{\tau_{\Lambda^{\prime}} Q \tau_{\Lambda} \tau_{N}}^{P\left(\mathbf{k}, k_{F}\right),} \\
\sum_{n(p)}^{\mathrm{GSC}}\left(\mathbf{k}, k_{F}\right)= & \sum_{P^{\prime}, P, Q, Q^{\prime}=D, E} \Gamma_{n(p)}^{P^{\prime} P Q Q^{\prime}}\left(\mathbf{k}, k_{F}\right)
\end{aligned}
$$

$$
\begin{aligned}
= & \sum_{P^{\prime}, P, Q, Q^{\prime}=D, E} \sum_{\tau_{N^{\prime}}, \tau_{\Lambda^{\prime}}, \tau_{\Lambda}, \tau_{N}=0,1} \mathcal{T}_{\tau_{N^{\prime}} \tau_{\Lambda^{\prime}} \tau_{\Lambda} \tau_{N}, n(p)}^{P^{\prime} P Q Q^{\prime}} \\
& \times \Gamma_{\tau_{N^{\prime}} \tau_{\Lambda^{\prime}} \tau_{\Lambda} \tau_{N}}^{P^{\prime} \tau_{N}}\left(\mathbf{k}, k_{F}\right),
\end{aligned}
$$

where $P^{\prime}, P, Q, Q^{\prime}=D$ or $E$ refer to the direct or exchange character of the matrix elements of Eq. (13). The isospin factors are given by

$$
\begin{aligned}
\mathcal{T}_{\tau_{\Lambda^{\prime}} \tau_{\Lambda}, n(p)}^{P Q}= & \sum_{f, \text { isospin }}\left\langle t_{\Lambda}\left|\mathcal{O}_{\tau_{\Lambda^{\prime}}}\right| f\right\rangle_{P}\left\langle f\left|\mathcal{O}_{\tau_{\Lambda}}\right| t_{\Lambda}\right\rangle_{Q}, \\
\mathcal{T}_{\tau_{\Lambda^{\prime}} \tau_{\Lambda} \tau_{N}, n(p)}^{P Q Q^{\prime}}= & \sum_{f, \text { isospin }}\left\langle t_{\Lambda}\left|\mathcal{O}_{\tau_{\Lambda^{\prime}}}\right| f\right\rangle_{P}\langle f| \\
& \times \mathcal{O}_{\tau_{\Lambda}}\left|t_{p} t_{h} t_{p^{\prime}} t_{h^{\prime}}, t_{\Lambda}\right\rangle_{Q} \\
& \times\left\langle t_{p} t_{h} t_{p^{\prime}} t_{h^{\prime}}, t_{\Lambda}\left|\mathcal{O}_{\tau_{N}}\right| t_{\Lambda}\right\rangle_{Q^{\prime}}, \\
\mathcal{T}_{\tau_{N^{\prime}} \tau_{\Lambda^{\prime}} \tau_{\Lambda} \tau_{N}, n(p)}^{P^{\prime} P Q^{\prime}}= & \sum_{f, \text { isospin }}\left\langle t_{\Lambda}\left|\mathcal{O}_{\tau_{N^{\prime}}}\right| t_{\tilde{p}} t_{\tilde{h}} t_{\tilde{p}^{\prime}} t_{\tilde{p}^{\prime}}, t_{\Lambda}\right\rangle_{P^{\prime}} \\
& \times\left\langle t_{\tilde{p}} t_{\tilde{h}} t_{\tilde{p}^{\prime}} t_{\tilde{p}^{\prime}}, t_{\Lambda}\left|\mathcal{O}_{\tau_{\Lambda^{\prime}}}\right| f\right\rangle_{P}\langle f| \mathcal{O}_{\tau_{\Lambda}} \\
& \times\left|t_{p} t_{h} t_{p^{\prime}} t_{h^{\prime}}, t_{\Lambda}\right\rangle_{Q}\left\langle t_{p} t_{h} t_{p^{\prime}} t_{h^{\prime}}, t_{\Lambda}\left|\mathcal{O}_{\tau_{N}}\right| t_{\Lambda}\right\rangle_{Q^{\prime}},
\end{aligned}
$$

where the summations run over all the isospin projections $t$ 's, with the constraint that the emitted particles are $n n$ for $\Gamma_{n}$ and $n p$ for $\Gamma_{p}$. For the partial decay widths we instead find

$$
\begin{aligned}
& \Gamma_{\tau_{\Lambda^{\prime}} \tau_{\Lambda}}^{P Q}\left(\mathbf{k}, k_{F}\right)=\mathcal{N}^{2}\left(k_{F}\right)(-1)^{n} \sum_{f} \delta\left(E_{f}-E_{0}\right) \\
& \times\left\langle p_{\Lambda}\left|\left[\mathcal{V}_{\tau_{\Lambda^{\prime}}}^{\Lambda N \rightarrow N N}\left(q^{\prime}\right)\right]^{\dagger}\right| f\right\rangle_{P}\langle f| \\
& \times \mathcal{V}_{\tau_{\Lambda}}^{\Lambda N \rightarrow N N}(q)\left|p_{\Lambda}\right\rangle_{Q} \text {, } \\
& \Gamma_{\tau_{\Lambda^{\prime}} \tau_{\Lambda} \tau_{N}}^{P Q Q^{\prime}}\left(\mathbf{k}, k_{F}\right)=-2 \mathcal{N}^{2}\left(k_{F}\right)(-1)^{n} \sum_{f} \sum_{p, h, p^{\prime}, h^{\prime}} \delta\left(E_{f}-E_{0}\right) \\
& \times\left\langle p_{\Lambda}\left|\left[\mathcal{V}_{\tau_{\Lambda^{\prime}}}^{\Lambda N \rightarrow N N}\left(q^{\prime}\right)\right]^{\dagger}\right| f\right\rangle_{P} \\
& \times\left\langle f\left|\mathcal{V}_{\tau_{\Lambda}}^{\Lambda N \rightarrow N N}(q)\right| p h p^{\prime} h^{\prime} ; p_{\Lambda}\right\rangle_{Q} \\
& \times \frac{\left\langle p h p^{\prime} h^{\prime} ; p_{\Lambda}\left|\mathcal{V}_{\tau_{N}}^{N N}(t)\right| p_{\Lambda}\right\rangle_{Q^{\prime}}}{\varepsilon_{p}-\varepsilon_{h}+\varepsilon_{p^{\prime}}-\varepsilon_{h^{\prime}}} \\
& \Gamma_{\tau_{N^{\prime}} \tau_{\Lambda^{\prime}} \tau_{\Lambda} \tau_{N}}^{P^{\prime} P Q Q^{\prime}}\left(\mathbf{k}, k_{F}\right)=\mathcal{N}^{2}\left(k_{F}\right)(-1)^{n} \sum_{f} \\
& \times \sum_{\tilde{p}, \tilde{h}, \tilde{p}^{\prime}, \tilde{h}^{\prime}} \sum_{p, h, p^{\prime}, h^{\prime}} \delta\left(E_{f}-E_{0}\right) \\
& \times \frac{\left\langle p_{\Lambda}\left|\left[\mathcal{V}_{\tau_{N^{\prime}}}^{N N}\left(t^{\prime}\right)\right]^{\dagger}\right| \tilde{p}, \tilde{h}, \tilde{p}^{\prime}, \tilde{h}^{\prime} ; p_{\Lambda}\right\rangle_{P^{\prime}}}{\varepsilon_{\tilde{p}}-\varepsilon_{\tilde{h}}+\varepsilon_{\tilde{p}^{\prime}}-\varepsilon_{\tilde{h}^{\prime}}} \\
& \times\left\langle\tilde{p}, \tilde{h}, \tilde{p}^{\prime}, \tilde{h}^{\prime} ; p_{\Lambda}\left|\left[\mathcal{V}_{\tau_{\Lambda^{\prime}}}^{\Lambda N \rightarrow N N}\left(q^{\prime}\right)\right]^{\dagger}\right| f\right\rangle_{P} \\
& \times\left\langle f\left|\mathcal{V}_{\tau_{\Lambda}}^{\Lambda N \rightarrow N N}(q)\right| p h p^{\prime} h^{\prime} ; p_{\Lambda}\right\rangle_{Q} \\
& \times \frac{\left\langle p h p^{\prime} h^{\prime} ; p_{\Lambda}\left|\mathcal{V}_{\tau_{N}}^{N N}(t)\right| p_{\Lambda}\right\rangle_{Q^{\prime}}}{\varepsilon_{p}-\varepsilon_{h}+\varepsilon_{p^{\prime}}-\varepsilon_{h^{\prime}}} \text {. }
\end{aligned}
$$

Note that the values of the energy momentum carried by the particle and hole lines depends on the topology of the corresponding diagram, while $n$ is the number of crossing between fermionic lines.

Let us now apply the preceding formalism to a model including amplitudes (a) and (b1) of Fig. 3. Four direct 


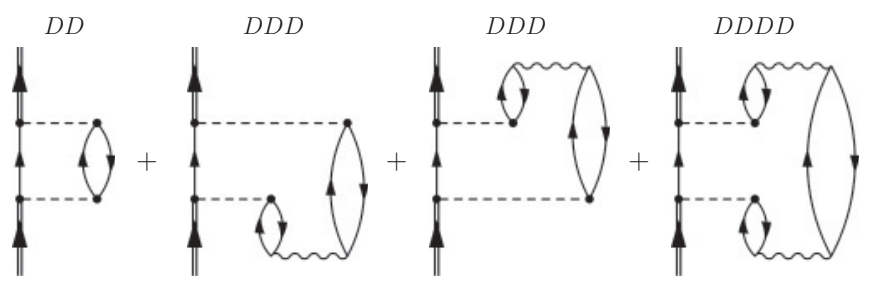

FIG. 6. Direct Goldstone diagrams corresponding to the square of the amplitude sum (a) + (b1) of Fig. 3. See the decomposition of Eq. (14).

self-energy diagrams correspond to the square of the amplitude sum (a) $+(b 1)$; they are given in Fig. 6. Note that these diagrams admit a single cut, giving rise to a $2 \mathrm{p} 1 \mathrm{~h}$ final state. The $D D$ diagram contributes to the partial widths $\Gamma_{n(p)}^{0}$ of Eq. (14). The two $D D D$ diagrams, which have the same numerical value and are interferences between amplitudes (a) and (b1) of Fig. 3, are included in the partial widths $\Gamma_{n(p)}^{0-\mathrm{GSC}}$. Finally, the diagram $D D D D$ contributes to $\Gamma_{n(p)}^{\mathrm{GSC}}$. Many exchange diagrams are obtained from the antisymmetrized amplitude sum (a) $+(\mathrm{b} 1)$ : One $P Q$ exchange diagram is the partner of the $D D$ one of Fig. 6; $7 P Q Q^{\prime}$ exchange diagrams are companions of each of the $D D D$ ones; $15 P^{\prime} P Q Q^{\prime}$ exchange diagrams add to the $D D D D$ one.

Formal expressions for $\Gamma_{n(p)}^{0}$ can be found in Ref. [20]. The $\Gamma_{n(p)}^{P Q Q^{\prime}}$, s contributing to $\Gamma_{n(p)}^{0-\mathrm{GSC}}$ [see Eq. (14)] correspond to the diagrams of Fig. 7. By replacing, in Eq. (16), the sum over momenta by integrals and by performing the energy integrations and the spin summation, the following expression for $\Gamma_{\tau_{\Lambda^{\prime}} \tau_{\Lambda} \tau_{N}}^{P Q Q^{\prime}}$ can be obtained:

$$
\begin{aligned}
\Gamma_{\tau_{\Lambda^{\prime}} \tau_{\Lambda} \tau_{N}}^{P Q Q^{\prime}}\left(\mathbf{k}, k_{F}\right)= & \mathcal{N}^{2}\left(k_{F}\right) \frac{1}{4} \frac{(-1)^{n}}{(2 \pi)^{8}}\left(G_{F} m_{\pi}^{2}\right)^{2} \frac{f_{\pi}^{2}}{m_{\pi}^{2}} \\
& \times \iiint d \mathbf{q} d \mathbf{h} d \mathbf{h}^{\prime} \mathcal{W}_{\tau_{\Lambda^{\prime}} \tau_{\Lambda} \tau_{N}}^{P Q Q^{\prime}}\left(q, q^{\prime}, t\right) \\
& \times \Theta\left(k, q, q^{\prime}, t, h, h^{\prime}, k_{F}\right) \frac{1}{-\varepsilon_{2 \mathrm{p} 2 \mathrm{~h}}^{P Q Q^{\prime}}} \\
& \times \delta\left[q_{0}-\left(\varepsilon_{\mathbf{h}^{\prime}+\mathbf{q}}-\varepsilon_{\mathbf{h}^{\prime}}\right)\right],
\end{aligned}
$$

where $q_{0}=k_{0}-\varepsilon_{\mathbf{k}-\mathbf{q}}-V_{N}, \quad V_{N}$ being the nucleonbinding energy, while the functions $\mathcal{W}_{\tau_{\Lambda^{\prime}} Q \tau_{\Lambda} \tau_{N}}^{P}\left(q, q^{\prime}, t\right)$ and $\Theta\left(k, q, q^{\prime}, t, h, h^{\prime}, k_{F}\right)$ and the energy denominator $\varepsilon_{2 p 2 h}^{P Q Q^{\prime}}$ are specific to each $P Q Q^{\prime}$ contribution. The function $\mathcal{W}_{\tau^{\prime}, \tau_{\wedge} \tau_{N}}^{P Q Q^{\prime}}\left(q, q^{\prime}, t\right)$ contains the momentum dependence of the nuclear residual interaction and the weak transition potentials and the spin summation, while $\Theta\left(k, q, q^{\prime}, t, h, h^{\prime}, k_{F}\right)$ is a product of step functions that define the phase space of particles and holes.

In the present section we present the explicit expression for the direct term $\Gamma_{\tau_{\Lambda^{\prime}} \tau_{\Lambda} \tau_{N}}^{D D D}$; the seven other ones are displayed in Appendix A. We obtain

$$
\begin{aligned}
\Gamma_{\tau_{\Lambda^{\prime}} \tau_{\Lambda} \tau_{N}}^{D D D}\left(\mathbf{k}, k_{F}\right)= & \mathcal{N}^{2}\left(k_{F}\right) \frac{1}{4} \frac{1}{(2 \pi)^{8}}\left(G_{F} m_{\pi}^{2}\right)^{2} \frac{f_{\pi}^{2}}{m_{\pi}^{2}} \\
& \times \iiint d \mathbf{q} d \mathbf{h} d \mathbf{h}^{\prime} \mathcal{W}_{\tau_{\Lambda^{\prime}} \tau_{\Lambda} \tau_{N}}^{2 D D}(q) \\
& \times \theta\left(q_{0}\right) \theta\left(|\mathbf{k}-\mathbf{q}|-k_{F}\right) \theta\left(|\mathbf{q}-\mathbf{h}|-k_{F} \mid\right)
\end{aligned}
$$


FIG. 7. Goldstone diagrams for the partial rates $\Gamma_{n(p)}^{P Q Q^{\prime}}$ contributing to Eq. (14).

$$
\begin{aligned}
& \times \theta\left(k_{F}-|\mathbf{h}|\right) \theta\left(\left|\mathbf{q}+\mathbf{h}^{\prime}\right|-k_{F} \mid\right) \theta\left(k_{F}-\left|\mathbf{h}^{\prime}\right|\right) \\
& \times \frac{1}{-q_{0}-\left(\varepsilon_{\mathbf{h}-\mathbf{q}}-\varepsilon_{\mathbf{h}}\right)} \delta\left[q_{0}-\left(\varepsilon_{\mathbf{h}^{\prime}+\mathbf{q}}-\varepsilon_{\mathbf{h}^{\prime}}\right)\right] .
\end{aligned}
$$

The expressions for $\Theta\left(k, q, q^{\prime}, t, h, h^{\prime}, k_{F}\right)$ and $\varepsilon_{2 p 2 h}^{D D D}$ are selfevident. Moreover,

$$
\begin{aligned}
& \mathcal{W}_{\tau_{\Lambda^{\prime}} \tau_{\Lambda} \tau_{N}}^{D D D}(q) \\
&= 8\left\{\left[S_{\tau_{\Lambda^{\prime}}^{\prime}}^{\prime}(q) S_{\tau_{\Lambda}}^{\prime}(q)+P_{C, \tau_{\Lambda^{\prime}}}(q) P_{C, \tau_{\Lambda}}(q)\right] \mathcal{V}_{C, \tau_{N}}(q)\right. \\
&+\left[S_{\tau_{\Lambda^{\prime}}}(q) S_{\tau_{\Lambda}}(q)+P_{L, \tau_{\Lambda^{\prime}}}(q) P_{L, \tau_{\Lambda}}(q)\right] \mathcal{V}_{L, \tau_{N}}(q) \\
&\left.+2\left[S_{V, \tau_{\Lambda^{\prime}}}(q) S_{V, \tau_{\Lambda}}(q)+P_{T, \tau_{\Lambda^{\prime}}}(q) P_{T, \tau_{\Lambda}}(q)\right] \mathcal{V}_{T, \tau_{N}}(q)\right\}
\end{aligned}
$$

Equation (19) can be simplified by introducing the functions

$$
\begin{aligned}
\mathcal{I}\left(q_{0}, \mathbf{q}\right)= & \frac{-\pi}{(2 \pi)^{3}} \int d \mathbf{h}^{\prime} \theta\left(\left|\mathbf{q}+\mathbf{h}^{\prime}\right|-k_{F} \mid\right) \\
& \times \theta\left(k_{F}-\left|\mathbf{h}^{\prime}\right|\right) \delta\left(q_{0}-\varepsilon_{\mathbf{h}^{\prime}+\mathbf{q}}+\varepsilon_{\mathbf{h}^{\prime}}\right),
\end{aligned}
$$




$$
\mathcal{R}\left(q_{0}, \mathbf{q}\right)=\frac{1}{(2 \pi)^{3}} \mathcal{P} \int d \mathbf{h} \frac{\theta\left(|\mathbf{q}-\mathbf{h}|-k_{F} \mid\right) \theta\left(k_{F}-|\mathbf{h}|\right)}{q_{0}-\left(\varepsilon_{\mathbf{h}-\mathbf{q}}-\varepsilon_{\mathbf{h}}\right)},
$$

where $\mathcal{I}\left(q_{0}, \mathbf{q}\right)$ is the imaginary part of the Lindhard function and the explicit expression for $\mathcal{R}\left(q_{0}, \mathbf{q}\right)$ is given in Appendix B. Therefore,

$$
\begin{aligned}
& \Gamma_{\tau_{\Lambda^{\prime}} \tau_{\Lambda} \tau_{N}}^{D D D}\left(\mathbf{k}, k_{F}\right) \\
&=-\frac{\mathcal{N}^{2}\left(k_{F}\right)}{(2 \pi)^{3}}\left(G_{F} m_{\pi}^{2}\right)^{2} \frac{f_{\pi}^{2}}{m_{\pi}^{2}} \int d \mathbf{q} \theta\left(q_{0}\right) \theta\left(|\mathbf{k}-\mathbf{q}|-k_{F}\right) \\
& \times\left\{\left[S_{\tau_{\Lambda^{\prime}}}^{\prime}(q) S_{\tau_{\Lambda}}^{\prime}(q)+P_{C, \tau_{\Lambda^{\prime}}}(q) P_{C, \tau_{\Lambda}}(q)\right] \mathcal{V}_{C, \tau_{N}}(q)\right. \\
&+\left[S_{\tau_{\Lambda^{\prime}}}(q) S_{\tau_{\Lambda}}(q)+P_{L, \tau_{\Lambda^{\prime}}}(q) P_{L, \tau_{\Lambda}}(q)\right] \mathcal{V}_{L, \tau_{N}}(q) \\
&\left.+2\left[S_{V, \tau_{\Lambda^{\prime}}}(q) S_{V, \tau_{\Lambda}}(q)+P_{T, \tau_{\Lambda^{\prime}}}(q) P_{T, \tau_{\Lambda}}(q)\right] \mathcal{V}_{T, \tau_{N}}(q)\right\} \\
& \times \mathcal{R}\left(-q_{0}, \mathbf{q}\right) \mathcal{I}\left(q_{0}, \mathbf{q}\right) .
\end{aligned}
$$

Then one has to perform the isospin summation to obtain

$$
\Gamma_{n(p)}^{D D D}\left(\mathbf{k}, k_{F}\right)=\sum_{\tau_{\Lambda^{\prime}}, \tau_{\Lambda}, \tau_{N}=0,1} \mathcal{T}_{\tau_{\Lambda^{\prime}}}^{P Q \tau_{\Lambda} \tau_{N}, n(p)} \Gamma_{\tau_{\Lambda^{\prime}} \tau_{\Lambda} \tau_{N}}^{D D D}\left(\mathbf{k}, k_{F}\right) .
$$

The final results obtained after the local-density approximation are therefore

$$
\begin{aligned}
& \Gamma_{n}^{D D D}=2\left\{\Gamma_{111}^{D D D}+\Gamma_{000}^{D D D}+\Gamma_{010}^{D D D}+\Gamma_{101}^{D D}\right\}, \\
& \Gamma_{p}^{D D D}=2\left\{5 \Gamma_{111}^{D D D}+\Gamma_{000}^{D D D}-\Gamma_{010}^{D D D}-\Gamma_{101}^{D D D}\right\} .
\end{aligned}
$$

Finally, we present the partial rates corresponding to diagram $D D D D$ of Fig. 6 . By applying the same procedure used for $\Gamma_{\tau_{\Lambda^{\prime}} \tau_{\Lambda} \tau_{N}}^{D D D D}$ to Eq. (17) we obtain

$$
\begin{aligned}
\Gamma_{\tau_{N^{\prime}} \tau_{\Lambda^{\prime}} \tau_{\Lambda} \tau_{N}}^{D D D}\left(\mathbf{k}, k_{F}\right)= & -\frac{\mathcal{N}^{2}\left(k_{F}\right)}{(2 \pi)^{2}}\left(G_{F} m_{\pi}^{2}\right)^{2}\left(\frac{f_{\pi}^{2}}{m_{\pi}^{2}}\right)^{2} \\
& \times \int d \mathbf{q} \theta\left(q_{0}\right) \theta\left(|\mathbf{k}-\mathbf{q}|-k_{F}\right) \\
& \times\left\{\left(S_{\tau_{\Lambda^{\prime}}}^{\prime} S_{\tau_{\Lambda}}^{\prime}+P_{C, \tau_{\Lambda^{\prime}}} P_{C, \tau_{\Lambda}}\right) \mathcal{V}_{C, \tau_{N}}^{2}\right. \\
& +\left(S_{\tau_{\Lambda^{\prime}}} S_{\tau_{\Lambda}}+P_{L, \tau_{\Lambda^{\prime}}} P_{L, \tau_{\Lambda}}\right) \mathcal{V}_{L, \tau_{N}}^{2} \\
& \left.+2\left(S_{V, \tau_{\Lambda^{\prime}}} S_{V, \tau_{\Lambda}}+P_{T, \tau_{\Lambda^{\prime}}} P_{T, \tau_{\Lambda}}\right) \mathcal{V}_{T, \tau_{N}}^{2}\right\} \\
& \times \mathcal{R}^{2}\left(-q_{0}, \mathbf{q}\right) \mathcal{I}\left(q_{0}, \mathbf{q}\right),
\end{aligned}
$$

and

$$
\begin{aligned}
& \Gamma_{n}^{D D D D}=4\left\{\Gamma_{1111}^{D D D}+\Gamma_{0000}^{D D D}+\Gamma_{0101}^{D D D}+\Gamma_{1010}^{D D D}\right\}, \\
& \Gamma_{p}^{D D D D}=4\left\{5 \Gamma_{1111}^{D D D}+\Gamma_{0000}^{D D D}-\Gamma_{0101}^{D D D}-\Gamma_{1010}^{D D D}\right\},
\end{aligned}
$$

after performing the local density approximation.

In this article the $\Gamma_{n(p)}^{P^{\prime} P Q Q^{\prime}}$ exchange terms will be neglected. Indeed, from our numerical results discussed in the next section it turns out that already the direct contribution $\Gamma_{n(p)}^{D D D}$ is small and approximately one order of magnitude smaller than $\Gamma_{n(p)}^{D D D}$. Moreover, according to the results obtained for the $\Gamma_{n(p)}^{P Q Q^{\prime}}$ s, $P^{\prime} P Q Q^{\prime}$ exchange contributions are expected to be even smaller than the $D D D D$ direct term.

\section{RESULTS}

In the previous section we have seen how the neutron- and proton-induced decay widths can be written in the form

$$
\begin{aligned}
\Gamma_{n(p)}= & \Gamma_{n(p)}^{0}+\Gamma_{n(p)}^{0-\mathrm{GSC}}+\Gamma_{n(p)}^{\mathrm{GSC}} \\
\equiv & \sum_{P, Q=D, E} \Gamma_{n(p)}^{P Q}+\sum_{P, Q, Q^{\prime}=D, E} \Gamma_{n(p)}^{P Q Q^{\prime}} \\
& +\sum_{P^{\prime}, P, Q, Q^{\prime}=D, E} \Gamma_{n(p)}^{P^{\prime} P Q Q^{\prime}},
\end{aligned}
$$

$\Gamma_{n(p)}^{0}$ being the rates obtained for an uncorrelated hypernuclear ground state, $\Gamma_{n(p)}^{\mathrm{GSC}}$ the rates originated by ground-state correlations and $\Gamma_{n(p)}^{0-G S C}$ the rates resulting from the interference between uncorrelated and correlated ground states.

For the present scheme containing the transition amplitudes (a) and (b1) of Fig. 3, where antisymmetrization is considered for the weak transition potential $V^{\Lambda N \rightarrow N N}$ and the nuclear residual interaction $V^{N N}$, we obtained 2 contributions to $\Gamma_{n(p)}^{0}$, which are $\Gamma_{n(p)}^{D D}=\Gamma_{n(p)}^{E E}$ and $\Gamma_{n(p)}^{D E}=\Gamma_{n(p)}^{E D}$ and are generated by the square of amplitude (a); 8 different $\Gamma_{n(p)}^{P Q Q^{\prime}}$ contributions to $\Gamma_{n(p)}^{0-\mathrm{GSC}}$, which are interferences between the (a) and (b1) amplitudes; 16 different $\Gamma_{n(p)}^{P^{\prime} P Q Q^{\prime}}$ contributions to $\Gamma_{n(p)}^{\mathrm{GSC}}$, which originate from the square of amplitude (b1). An early evaluation of $\Gamma_{n(p)}^{0}$ has been performed in Ref. [20], while $\Gamma_{n(p)}^{0-\mathrm{GSC}}$ and $\Gamma_{n(p)}^{\mathrm{GSC}}$ are discussed here for the first time. Among the $\Gamma_{n(p)}^{P^{\prime} P Q Q^{\prime}}$,s, here we only calculate the direct terms $\Gamma_{n(p)}^{D D D D}$.

\section{A. ${ }_{\Lambda}^{12} \mathrm{C}$}

We start by discussing the relevance of the Pauli exchange terms in $\Gamma_{n(p)}^{0}$ and $\Gamma_{n(p)}^{0-\mathrm{GSC}}$. Our results for $\Gamma_{n}^{P Q}$ and $\Gamma_{p}^{P Q}$ are given in Table I for the decay of ${ }_{\Lambda}^{12} \mathrm{C}$. Note that, for symmetry, $\Gamma_{n(p)}^{0}$ are twice the sum of $\Gamma_{n(p)}^{D D}$ and $\Gamma_{n(p)}^{D E}$. Exchange terms contribute to the uncorrelated rates for neutron-induced (proton-induced) decays by $5.1 \%(0.3 \%)$. Thus, they tend to increase $\Gamma_{n} / \Gamma_{p}$ while having a very small effect on $\Gamma_{1}$.

In Table II we present predictions for the $\Gamma_{n}^{P Q Q^{\prime}}$ and $\Gamma_{p}^{P Q Q^{\prime}}$ contributions derived from the Goldstone diagrams of Fig. 7, again for ${ }_{\Lambda}^{12} \mathrm{C}$. As expected, the direct terms $\Gamma_{n}^{D D D}$ and $\Gamma_{p}^{D D D}$ are the main contributions. Nevertheless, the effect of antisymmetry on the two isospin channels is significant: It increases $\Gamma_{n}^{0-\mathrm{GSC}}$ by $34 \%$ while decreasing $\Gamma_{p}^{0-\mathrm{GSC}}$ by $8 \%$. The overall effect on $\Gamma_{1}^{0-\mathrm{GSC}}=\Gamma_{n}^{0-\mathrm{GSC}}+\Gamma_{p}^{0-\mathrm{GSC}}$ is a very small

TABLE I. Direct and exchange $\Gamma_{n}^{P Q}$ and $\Gamma_{p}^{P Q}$ terms for ${ }_{\Lambda}^{12} \mathrm{C}$ in units of the free $\Lambda$-decay rate, $\Gamma^{0}=2.52 \times 10^{-6} \mathrm{eV}$. The first column indicates the two different isospin channels and their sum. Note that $\Gamma_{n(p)}^{D D}=\Gamma_{n(p)}^{E E}$ and $\Gamma_{n(p)}^{D E}=\Gamma_{n(p)}^{E D}$.

\begin{tabular}{llll}
\hline \hline Channel & $2 \Gamma^{D D}$ & $2 \Gamma^{D E}$ & $\Gamma^{0}$ \\
\hline$\Lambda n \rightarrow n n$ & 0.146 & 0.008 & 0.154 \\
$\Lambda p \rightarrow n p$ & 0.469 & 0.002 & 0.470 \\
Sum & 0.615 & 0.009 & 0.624 \\
\hline \hline
\end{tabular}


TABLE II. Direct and exchange $\Gamma_{n}^{P Q Q^{\prime}}$ and $\Gamma_{p}^{P Q Q^{\prime}}$ terms for ${ }_{\Lambda}^{12} \mathrm{C}$ obtained from the diagrams of Fig. 7.

\begin{tabular}{lccccc}
\hline \hline Channel & $\Gamma^{D D D}$ & $\Gamma^{D D E}$ & $\Gamma^{D E D}$ & $\Gamma^{E D D}$ & \\
\hline$\Lambda n \rightarrow n n$ & 0.022 & -0.002 & -0.009 & -0.004 & \\
$\Lambda p \rightarrow n p$ & 0.071 & 0.005 & -0.027 & -0.011 & \\
Sum & 0.093 & 0.003 & -0.036 & -0.015 & \\
& $\Gamma^{D E E}$ & $\Gamma^{E D E}$ & $\Gamma^{E E D}$ & $\Gamma^{E E E}$ & $\Gamma^{0-\mathrm{GSC}}$ \\
$\Lambda n \rightarrow n n$ & 0.006 & 0.008 & 0.006 & 0.002 & 0.029 \\
$\Lambda p \rightarrow n p$ & -0.008 & 0.009 & 0.025 & 0.002 & 0.066 \\
Sum & -0.003 & 0.017 & 0.031 & 0.004 & 0.095 \\
\hline \hline
\end{tabular}

increase, of $2 \%$. We note that, with topologically equivalent diagrams, in Ref. [24] a similar quasicancellation between neutron- and proton-induced decays has been found in nucleon spectra calculations. Moreover, in Ref. [18] it has been shown that the evaluation of the GSC exchange terms is important for the rate $\Gamma_{2}$ as well. We emphasize that the exact evaluation of exchange diagrams has been mostly ignored in the literature. It is usually a quite involved (but necessary) task, given the rapidly increasing number of terms one has to consider when going to higher orders in the nuclear residual interaction. Unfortunately, there is no general rule for anticipating the need for the evaluation of exchange terms when the corresponding direct contribution is important.

In Table III we present the different contributions to the rates $\Gamma_{n}$ and $\Gamma_{p}$ of Eq. (28). The uncorrelated parts $\Gamma_{n}^{0}$ and $\Gamma_{p}^{0}$ dominate over the remaining ones: $\Gamma_{1}^{0}=\Gamma_{n}^{0}+\Gamma_{p}^{0}$ constitutes $86 \%$ of the total $\Gamma_{1}$. Then, $\Gamma_{1}^{0-\mathrm{GSC}}=\Gamma_{n}^{0-\mathrm{GSC}}+$ $\Gamma_{p}^{0-\mathrm{GSC}}$ and $\Gamma_{1}^{\mathrm{GSC}}=\Gamma_{n}^{\mathrm{GSC}}+\Gamma_{p}^{\mathrm{GSC}}$ represent $13 \%$ and $1 \%$ of $\Gamma_{1}$, respectively. We remind the reader that $\Gamma_{n(p)}^{\mathrm{GSC}}$ are calculated from the direct diagram $D D D D$ in Fig. 6, while $P^{\prime} P Q Q^{\prime}$ exchange terms are neglected. This omission is justified by the smallness of the direct contributions $\Gamma_{n(p)}^{D D D D}$ : The neglected exchange part of $\Gamma_{1}^{\mathrm{GSC}}$ should contribute to $\Gamma_{1}$ by less than $1 \%$. Thus, a challenging calculation of the $15 P^{\prime} P Q Q^{\prime}$ exchange diagrams can be reasonably avoided.

Our predictions for the one- and two-nucleon induced decay rates for ${ }_{\Lambda}^{12} \mathrm{C}$ are given in Table IV. For completeness, we report results without and with the inclusion of antisymmetrization and GSCs. It should be noted that the hypernuclear groundstate normalization function $\mathcal{N}\left(k_{F}\right)$ of Eq. (5) equally affects $\Gamma_{1}$ and $\Gamma_{2}$. This function is not identically equal to one only when GSCs are present. Therefore, the $\Gamma_{1}$ result without GSC and with exchange terms of Table IV, 0.74 , is bigger than the prediction for $\Gamma_{1}^{0}$ of Table III, 0.62 , which has been obtained instead by including both GSCs and antisymmetrization in

TABLE III. Predictions for the one-nucleon induced decay rates of Eq. (28) for ${ }_{\Lambda}^{12} \mathrm{C}$.

\begin{tabular}{lcccc}
\hline \hline Channel & $\Gamma^{0}$ & $\Gamma^{0-\mathrm{GSC}}$ & $\Gamma^{\mathrm{GSC}}$ & $\Gamma$ \\
\hline$\Lambda n \rightarrow n n$ & 0.154 & 0.029 & 0.002 & 0.185 \\
$\Lambda p \rightarrow n p$ & 0.470 & 0.066 & 0.008 & 0.544 \\
Sum & 0.624 & 0.095 & 0.010 & 0.729 \\
\hline \hline
\end{tabular}

the normalization function. This comparison gives an idea of the importance of a proper normalization of the hypernuclear ground state. GSCs produce a sizable increase in the value of $\Gamma_{\mathrm{NM}}$, thanks to the opening of the two-nucleon induced channel, while $\Gamma_{1}$ remains practically unaffected. The effect of GSCs on the $\Gamma_{n} / \Gamma_{p}$ ratio is a small increase of $4 \%$, which is attributable entirely to the exchange terms in $\Gamma_{n}^{0-\text { GSC }}$ and $\Gamma_{p}^{0-\mathrm{GSC}}$ (see Table II). Antisymmetrization, on the contrary, introduces an increase of $\Gamma_{1}$ and a reduction of $\Gamma_{2}$ and as a result a sizable reduction of $\Gamma_{2} / \Gamma_{1}$.

In Table IV our predictions are compared with the most recent data by KEK [31] and FINUDA [32]. It must be noted that the partial decay rates of both data sets were obtained by deconvoluting the effect of nucleon rescattering from the measured nucleon spectra, as discussed in Sec. II. From this comparison we conclude that GSCs are important for getting agreement with data on $\Gamma_{\mathrm{NM}}$, while antisymmetrization is crucial for reproducing the data for $\Gamma_{2} / \Gamma_{\mathrm{NM}}$ : Only with the set of results including both exchange terms and GSCs can we achieve an overall agreement with all data. This agreement leads us to believe that the effect of final-state nucleon-nucleon correlations on the decay rates, which we have not included in the calculation but that can in principle affect the data, is of limited size.

Nevertheless, we have to admit that more refined and systematic theoretical studies should be performed before one can reach definite conclusions from the comparison between theory and experiment. For instance, the result obtained for $\Gamma_{\mathrm{NM}}$ requires a comment on the eventual inclusion of the full set of diagrams stemming from the amplitudes in Figs. 3 and 4 and eventually from other amplitudes. At first glance, one may think that the final outcome from all these diagrams would be a bigger value for $\Gamma_{\mathrm{NM}}$, thus spoiling the good agreement with data of the present result. This is not necessarily the case, for two reasons. First, amplitudes (d1) and (d2) in Fig. 3 and amplitude (c) in Fig. 4 originate from the $1 \Delta 1 \mathrm{p} 2 \mathrm{~h}$ GSC. The inclusion of these correlation amplitudes requires the introduction of new terms in the ground-state normalization function (5); this leads to a reduction of the individual values for each decay width, including the ones we have obtained in the preceding. From the previous studies in Refs. [17,18] one observes the following property, introduced by ground-state normalization: A certain redistribution of the total nonmesonic decay strength among the partial contributions occurs when new self-energy terms are included. Second, the presence of several additional self-energy diagrams which are interference terms between different amplitudes could also bring a reduction of the decay rates $\Gamma_{1}$ and $\Gamma_{2}$.

\section{B. Medium and heavy hypernuclei}

To have a further indication of the reliability of our framework, which adopts the local density approximation to obtain results for finite hypernuclei, we have extended the calculation to medium and heavy $\Lambda$ hypernuclei. All the GSC contributions and the antisymmetrization terms discussed in detail for ${ }_{\Lambda}^{12} \mathrm{C}$ have been taken into account. The results we have obtained are given in Table $\mathrm{V}$ and are compared with recent data in Fig. 8. 
TABLE IV. The nonmesonic weak decay widths of ${ }_{\Lambda}^{12} \mathrm{C}$. Results are given without and with the contributions of antisymmetrization and ground-state correlations. The most recent data, from KEK [31] and FINUDA [32], are given for comparison.

\begin{tabular}{|c|c|c|c|c|c|c|c|}
\hline $\mathrm{No} / \mathrm{No}$ & 0.15 & 0.47 & 0.62 & 0 & 0.62 & 0.31 & 0 \\
\hline Yes/No & 0.18 & 0.56 & 0.74 & 0 & 0.74 & 0.33 & 0 \\
\hline Yes/Yes & 0.19 & 0.55 & 0.73 & 0.25 & 0.98 & 0.34 & 0.26 \\
\hline KEK & $0.23 \pm 0.08$ & $0.45 \pm 0.10$ & $0.68 \pm 0.13$ & $0.27 \pm 0.13$ & $0.95 \pm 0.04$ & $0.51 \pm 0.13 \pm 0.05$ & $0.29 \pm 0.13$ \\
\hline
\end{tabular}

The GSC-free rate $\Gamma_{1}^{0}$ represents $86 \%$ of the rate $\Gamma_{1}=$ $\Gamma_{1}^{0}+\Gamma_{1}^{0-\mathrm{GSC}}+\Gamma_{1}^{\mathrm{GSC}}$ for ${ }_{\Lambda}^{12} \mathrm{C}$. For increasing hypernuclear mass number $A$, this contribution decreases and reaches $81 \%$ for ${ }_{\Lambda}^{208} \mathrm{~Pb}$. As expected, GSC contributions are thus more important for heavy hypernuclei.

The one- and two-nucleon induced rates increase with $A$ and rapidly saturate. Saturation is expected to begin for those hypernuclei whose radius becomes sensitively larger than the range of the nonmesonic processes. The fact that for ${ }_{\Lambda}^{40} \mathrm{Ca}$ and ${ }_{\Lambda}^{208} \mathrm{~Pb}$ we obtain very similar predictions informs us that in ${ }_{\Lambda}^{208} \mathrm{~Pb}$ the nonmesonic decay (both one- and two-nucleon stimulated) involves the same nucleon shells that participate in the decay of ${ }_{\Lambda}^{40} \mathrm{Ca}$. Indeed, the $\Lambda$ wave function ( $s$ level of the $\Lambda$-nucleus mean potential) is well overlapped to the hypernuclear core already in ${ }_{\Lambda}^{40} \mathrm{Ca}$.

It should be noted that the slight decrease of the nonmesonic rate $\Gamma_{\mathrm{NM}}$ going from ${ }_{\Lambda}^{89} \mathrm{Y}$ to ${ }_{\Lambda}^{139} \mathrm{La}$ is attributable to the special value of the oscillator parameter $\hbar \omega$ adopted for this hypernucleus. Such a parameter, which is obtained as the difference between the measured $s$ and $p \Lambda$ energy levels in ${ }_{\Lambda}^{139} \mathrm{La}$, is indeed smaller than the values measured for the two neighboring hypernuclei of our calculation, ${ }_{\Lambda}^{89} \mathrm{Y}$ and ${ }_{\Lambda}^{208} \mathrm{~Pb}$.

The contribution of the two-nucleon induced width is almost independent of the hypernuclear mass number and oscillates between $22 \%$ and $26 \%$ of $\Gamma_{\mathrm{NM}}$. We note from Fig. 8 that the datum recently determined at $\mathrm{KEK}, \Gamma_{2}\left({ }_{\Lambda}^{12} \mathrm{C}\right)=$ $0.27 \pm 0.13$ [31] is well reproduced by our calculation. Also, the recent determination obtained by FINUDA [32] of $\Gamma_{2} / \Gamma_{\mathrm{NM}}=0.22 \pm 0.08$ for hypernuclei from ${ }_{\Lambda}^{5} \mathrm{He}$ to ${ }_{\Lambda}^{16} \mathrm{O}$ is in agreement with our predictions.

TABLE V. Decay rates predicted for medium to heavy hypernuclei.

\begin{tabular}{lcccc}
\hline \hline Hypernucleus & $\Gamma_{1}^{0}$ & $\Gamma_{1}$ & $\Gamma_{2}$ & $\Gamma_{\mathrm{NM}}$ \\
\hline${ }_{\Lambda}^{11} \mathrm{~B}$ & 0.56 & 0.64 & 0.18 & 0.82 \\
${ }_{\Lambda}^{12} \mathrm{C}$ & 0.62 & 0.73 & 0.25 & 0.98 \\
${ }_{\Lambda}^{27} \mathrm{Al}$ & 0.80 & 0.94 & 0.28 & 1.22 \\
${ }_{\Lambda}^{28} \mathrm{Si}$ & 0.81 & 0.96 & 0.29 & 1.25 \\
${ }_{\Lambda}^{40} \mathrm{Ca}$ & 0.87 & 1.03 & 0.29 & 1.33 \\
${ }_{\Lambda}^{56} \mathrm{Fe}$ & 0.88 & 1.06 & 0.33 & 1.39 \\
${ }_{\Lambda}^{89} \mathrm{Y}$ & 0.87 & 1.06 & 0.33 & 1.39 \\
${ }_{\Lambda}^{139} \mathrm{La}$ & 0.86 & 1.04 & 0.32 & 1.36 \\
${ }_{\Lambda}^{208} \mathrm{~Pb}$ & 0.86 & 1.06 & 0.34 & 1.40 \\
\hline \hline
\end{tabular}

Concerning $\Gamma_{\mathrm{NM}}$, the agreement of our predictions with data is also rather good. The only exception is the large underestimation of the datum for the $A \simeq 200$ region, which, however, is also difficult to reconcile with the decay rate measured at KEK for ${ }_{\Lambda}^{56} \mathrm{Fe}$. No known mechanism can be responsible for a large increase in the nonmesonic decay rate when going from ${ }_{\Lambda}^{56} \mathrm{Fe}$ to the $A \simeq 200$ region. Concerning the datum for $A \simeq 200$, we have to note that, given the difficulty in employing direct timing methods for heavy hypernuclei, it has been obtained in experiments (performed at COSY, Juelich [33]) that measured the fission fragments (which are supposed to be generated by the nonmesonic decay) emitted by hypernuclei produced in proton-nucleus reactions. Large uncertainties affect such delayed fission experiments because of the limited precision of the employed recoil shadow method. The produced hypernuclei cannot be unambiguously identified with this method. It is also possible that mechanisms other than the nonmesonic decay contributed to hypernuclear fission in these experiments. The datum reported in Fig. 8 has been obtained as an average from measurements for hypernuclei produced in proton- $\mathrm{Au}$, proton- $\mathrm{Bi}$, and proton- $\mathrm{U}$ reactions.

We think that the results of the evaluation for medium and heavy hypernuclei are encouraging: They give us some

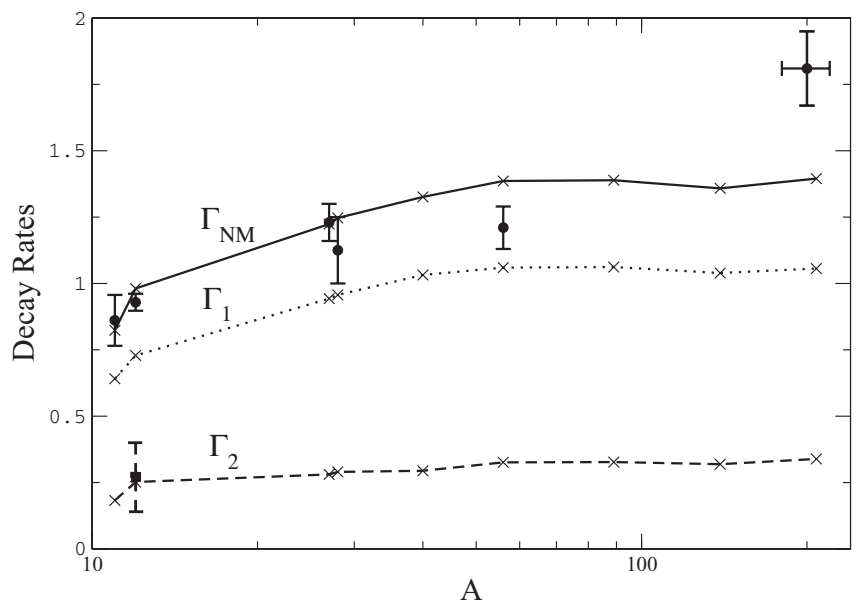

FIG. 8. The predictions for the decay rates $\Gamma_{1}, \Gamma_{2}$, and $\Gamma_{\mathrm{NM}}=$ $\Gamma_{1}+\Gamma_{2}$ are given as a function of the hypernuclear mass number $A$. The results for $\Gamma_{\mathrm{NM}}$ are compared with experimental data for ${ }_{\Lambda}^{11} \mathrm{~B}$ [34], ${ }_{\Lambda}^{12} \mathrm{C}$ [35], ${ }_{\Lambda}^{27} \mathrm{Al}$ [34], ${ }_{\Lambda}^{28} \mathrm{Si}$ [34], ${ }_{\Lambda}^{56} \mathrm{Fe}$ [34], and the region of $A$ between 180 and 220 [33]. The datum for $\Gamma_{2}$ is from Ref. [31]. 
confidence in using the local density approximation for obtaining results in finite hypernuclei, even in light systems such as ${ }_{\Lambda}^{12} \mathrm{C}$.

\section{Closing remarks}

Before concluding, we make here some further comments on our calculation. Through our work we wish to emphasize the importance of a detailed many-body treatment of nonmesonic decay. This requires the identification and evaluations of a large number of diagrams, working on a step-by-step basis with the perspective of reaching the condition in which the terms that are not taken into account can be safely neglected. Considering the evolution in the predictions obtained in recent works (see especially Refs. [17,18]) and here, this stability of results has not been achieved yet, and new many-body terms must be considered. In our opinion, one should explore the dependencies of predictions on the weak transition potential model only after these complicated many-body aspects are properly understood. Finally, one should attempt to reach a detailed agreement with experiment for $\Gamma_{\mathrm{NM}}, \Gamma_{n} / \Gamma_{p}$, and $\Gamma_{2} / \Gamma_{\mathrm{NM}}$ and thus extract sensible information on strangenesschanging baryon interactions. From the experimental side, new and improved data are expected from FINUDA@Daphne [36], JPARC [37,38], and GSI [39]. Questions of particular importance will be a direct experimental identification of the two-nucleon induced channels together with the determination of their contributions to the measured nucleon spectra.

We end this section with a comment to emphasize the importance of evaluating exchange terms. In our many-body inspired calculation, such terms are considered together with GSC contributions, which are included on the same ground for one- and two-nucleon induced decays. GSC and exchange terms improve by $10 \%$ the value of $\Gamma_{n} / \Gamma_{p}$. Once GSCs are included, antisymmetrization turns out to be particularly important for both the one- and the two-nucleon induced channels, reducing $\Gamma_{2}$ by $18 \%$ and increasing $\Gamma_{1}$ by $20 \%$. It would thus be pointless to neglect exchange terms and evaluate only direct ones. Although the introduction of antisymmetry is a difficult task in a many-body framework, one should evaluate all those exchange diagrams that are companions of a direct diagram that one knows to be relevant.

\section{CONCLUSIONS}

In this contribution we have studied the effects of GSCs in the nonmesonic weak decay of $\Lambda$ hypernuclei. A nonrelativistic nuclear-matter scheme has been adopted together with the local-density approximation for calculations in hypernuclei ranging from ${ }_{\Lambda}^{11} \mathrm{~B}$ to ${ }_{\Lambda}^{208} \mathrm{~Pb}$. All isospin channels contributing to one- and two-nucleon induced decays have been considered. The employed weak transition potential contains the exchange of mesons of the pseudoscalar and vector octets $\pi, \eta, K, \rho, \omega$, and $K^{*}$. The residual strong interaction, responsible for GSCs, has been modeled on a Bonn potential based on $\pi, \rho, \sigma$, and $\omega$ exchange.

By using the Goldstone diagrams technique, GSCs have been introduced on the same footing for one- and two-nucleon stimulated decays. The normalization of the hypernuclear ground state introduced by GSCs has been taken into account. We have devoted particular attention to those GSCs affecting the decay widths $\Gamma_{n}$ and $\Gamma_{p}$. The many-body $\Lambda$ self-energy terms we have considered originate from transition amplitudes (a) and (b1) of Fig. 3 (for one-nucleon induced decays) and by amplitude (a) of Fig. 4 (for two-nucleon induced decays). Our approach embodies fermion antisymmetry; that is, both direct and exchange interactions are considered in the various diagrams. Concerning one-nucleon induced decays, we have evaluated GSC-free rates $\Gamma_{n(p)}^{0}$, generated by amplitude 3(a), purely GSC terms $\Gamma_{n(p)}^{\mathrm{GSC}}$, produced by amplitude 3(b1), and interference terms $\Gamma_{n(p)}^{0-G S C}$ between uncorrelated and correlated hypernuclear ground states, that is, between amplitudes 3(a) and 3(b1).

The dominant contribution to $\Gamma_{1}=\Gamma_{1}^{0}+\Gamma_{1}^{0-\mathrm{GSC}}+\Gamma_{1}^{\mathrm{GSC}}$ turned out to be $\Gamma_{1}^{0}=\Gamma_{n}^{0}+\Gamma_{p}^{0}$. For ${ }_{\Lambda}^{12} \mathrm{C}, \Gamma_{1}^{0-\mathrm{GSC}}=\Gamma_{n}^{0-\mathrm{GSC}}+$ $\Gamma_{p}^{0-\mathrm{GSC}}$ and $\Gamma_{1}^{\mathrm{GSC}}=\Gamma_{n}^{\mathrm{GSC}}+\Gamma_{p}^{\mathrm{GSC}}$ represented $13 \%$ and $1 \%$ of the rate $\Gamma_{1}$, respectively; GSCs are thus responsible for $14 \%$ of the one-nucleon induced width (such contribution increases up to $19 \%$ for ${ }_{\Lambda}^{208} \mathrm{~Pb}$ ). The preceding results justify the fact that we have neglected the exchange terms in $\Gamma_{n(p)}^{\mathrm{GSC}}$. Exchange contributions are rather relevant in the calculation of $\Gamma_{n(p)}^{0-\mathrm{GSC}}$ (for ${ }_{\Lambda}^{12} \mathrm{C}$, they increase $\Gamma_{n}^{0-\mathrm{GSC}}$ by $34 \%$ and decreases $\Gamma_{p}^{0-\mathrm{GSC}}$ by $8 \%$ ), while only scarcely contribute to $\Gamma_{n(p)}^{0}$. GSCs and exchange terms together increase the value of $\Gamma_{n} / \Gamma_{p}$ for ${ }_{\Lambda}^{12} \mathrm{C}$ by $10 \%$. Thanks to the opening of the two-nucleon induced channel, GSC produces a sizable increase (of $32 \%$ for ${ }_{\Lambda}^{12} \mathrm{C}$ when exchange terms are included) in the value of $\Gamma_{\mathrm{NM}}=$ $\Gamma_{1}+\Gamma_{2}$.

The agreement among our final results and recent data is quite good and clearly demonstrates the necessity of including GSC and antisymmetrization effects. Nevertheless, we believe that a refinement of the present scheme must be pursued. Additional many-body terms should be considered, involving for instance the $\Delta(1232)$ resonance. Only after a certain stability of predictions is reached within such a microscopic approach should one explore the dependencies on the weak transition potential model and determine, through detailed comparison with experiment, sensible information on strangeness-changing baryon interactions.

\section{ACKNOWLEDGMENTS}

This work has been partially supported by the CONICET, under Contract No. PIP 6159. This research is part of the EU Initiative FP7-Project HadronPhysics2 under Project No. 227431. We thank A. Ramos and F. Krmpotic for the helpful discussion and the careful reading of the manuscript.

\section{APPENDIX A}

In this appendix we present explicit expressions for the decay rates $\Gamma_{n(p)}^{P Q Q^{\prime}}$ with $P Q Q^{\prime} \neq D D D$ associated with the Goldstone diagrams of Fig. 7 and contributing to Eq. (14). In 
the main text, these widths have been written as

$$
\Gamma_{n(p)}^{P Q Q^{\prime}}=\sum_{\tau_{\Lambda^{\prime}}, \tau_{\Lambda}, \tau_{N}=0,1} \mathcal{T}_{\tau_{\Lambda^{\prime}} \tau_{\Lambda} \tau_{N}, n(p)}^{P Q Q^{\prime}} \Gamma_{\tau_{\Lambda^{\prime}} \tau_{\Lambda} \tau_{N}}^{P Q Q^{\prime}}\left(\mathbf{k}, k_{F}\right)
$$

where

$$
\begin{aligned}
\Gamma_{\tau_{\Lambda^{\prime}} \tau_{\Lambda} \tau_{N}}^{P Q Q^{\prime}}\left(\mathbf{k}, k_{F}\right)= & \mathcal{N}^{2}\left(k_{F}\right) \frac{1}{4} \frac{(-1)^{n}}{(2 \pi)^{8}}\left(G_{F} m_{\pi}^{2}\right)^{2} \frac{f_{\pi}^{2}}{m_{\pi}^{2}} \\
& \times \iiint d \mathbf{q} d \mathbf{h} d \mathbf{h}^{\prime} \mathcal{W}_{\tau_{\Lambda^{\prime}} \tau_{\Lambda} \tau_{N}}^{P Q \tau^{\prime}}\left(q, q^{\prime}, t\right) \\
& \times \Theta\left(k, q, q^{\prime}, t, h, h^{\prime}, k_{F}\right) \frac{1}{-\varepsilon_{2 \mathrm{p} 2 \mathrm{~h}}^{P Q Q^{\prime}}} \\
& \times \delta\left[q_{0}-\left(\varepsilon_{\mathbf{h}^{\prime}+\mathbf{q}}-\varepsilon_{\mathbf{h}^{\prime}}\right)\right]
\end{aligned}
$$

The isospin index $\tau_{\Lambda}\left(\tau_{\Lambda^{\prime}}\right)$ of the weak transition potential is associated to an energy-momentum $q\left(q^{\prime}\right)$, while the nuclear strong interaction isospin index is $\tau_{N}$ and the corresponding energy-momentum $t$. In the following subsections we give the functions $\mathcal{W}_{\tau_{\Lambda^{\prime}} \tau_{\Lambda} \tau_{N}}^{P Q Q^{\prime}}\left(q, q^{\prime}, t\right)$ and $\Theta\left(k, q, q^{\prime}, t, h, h^{\prime}, k_{F}\right)$, the energy denominator $\varepsilon_{2 \mathrm{p} 2 \mathrm{~h}}^{P Q Q^{\prime}}$, and $n$ (the number of crossing between fermionic lines) for the various cases. Finally, we show the isospin sums of Eq. (A1).

\section{1. $\Gamma_{\mathbf{n}(\mathbf{p})}^{\mathrm{DDE}}$}

The $\mathcal{W}_{\tau_{\Lambda^{\prime}} \tau_{\Lambda} \tau_{N}}^{D D E}\left(q, q^{\prime}, t\right)$ function, where $q^{\prime}=q$ and $t=h^{\prime}-$ $h+q$, is identical to the $\mathcal{S}_{\tau^{\prime} \tau_{N} \tau}^{\text {ded }}\left(q, q^{\prime}, t\right)$ function in Eq. (A1) of Ref. [24]. Moreover,

$$
\begin{aligned}
\Theta( & \left.k, q, q^{\prime}, t, h, h^{\prime}, k_{F}\right) \\
= & \theta\left(q_{0}\right) \theta\left(|\mathbf{k}-\mathbf{q}|-k_{F}\right) \theta\left(|\mathbf{q}-\mathbf{h}|-k_{F} \mid\right) \\
& \times \theta\left(k_{F}-|\mathbf{h}|\right) \theta\left(\left|\mathbf{q}+\mathbf{h}^{\prime}\right|-k_{F} \mid\right) \theta\left(k_{F}-\left|\mathbf{h}^{\prime}\right|\right), \\
\varepsilon_{2 \mathrm{p} 2 \mathrm{~h}}^{D D E}= & \varepsilon_{2 \mathrm{p} 2 \mathrm{~h}}^{D D D} \equiv k_{0}-\varepsilon_{k}-q+\varepsilon_{h}-q-\varepsilon_{h}-V_{N},
\end{aligned}
$$

and $n=0$. The isospin sums are given by

$$
\begin{aligned}
\Gamma_{n}^{D D E}= & -\Gamma_{111}^{D D E}+\Gamma_{000}^{D D E}+3 \Gamma_{101}^{D D E}+\Gamma_{110}^{D D E}-\Gamma_{011}^{D D E} \\
& +\Gamma_{100}^{D D E}+3 \Gamma_{001}^{D D E}+\Gamma_{010}^{D D E}, \\
\Gamma_{p}^{D D E}= & -5 \Gamma_{111}^{D D E}+\Gamma_{000}^{D D E}-3 \Gamma_{101}^{D D E}+5 \Gamma_{110}^{D D E}+\Gamma_{011}^{D D E} \\
& -\Gamma_{100}^{D D E}+3 \Gamma_{001}^{D D E}-\Gamma_{010}^{D D E} .
\end{aligned}
$$

$$
\text { 2. } \Gamma_{\mathbf{n}(\mathbf{p})}^{\mathrm{DED}}
$$

The $\mathcal{W}_{\tau_{\Lambda^{\prime}} \tau_{\Lambda} \tau_{N}}^{\mathrm{DED}}\left(q, q^{\prime}, t\right)$ function, where $q^{\prime}=k-h$ and $t=$ $q$, is identical to the $\mathcal{S}_{\tau^{\prime} \tau_{N} \tau}^{\text {dde }}\left(q, q^{\prime}, t\right)$ function in Eq. (A3) of Ref. [24]. Moreover,

$$
\begin{aligned}
\Theta\left(k, q, q^{\prime}, t, h, h^{\prime}, k_{F}\right) & \\
= & \theta\left(q_{0}\right) \theta\left(|\mathbf{k}-\mathbf{q}|-k_{F}\right) \theta\left(|\mathbf{q}-\mathbf{h}|-k_{F} \mid\right) \\
& \times \theta\left(k_{F}-|\mathbf{h}|\right) \theta\left(\left|\mathbf{q}+\mathbf{h}^{\prime}\right|-k_{F} \mid\right) \theta\left(k_{F}-\left|\mathbf{h}^{\prime}\right|\right), \\
\varepsilon_{2 \mathrm{p} 2 \mathrm{~h}}^{D E D}= & \varepsilon_{2 \mathrm{p} 2 \mathrm{~h}}^{D D D},
\end{aligned}
$$

and $n=0$. The isospin sums are given by

$$
\begin{aligned}
\Gamma_{n}^{D E D}= & -\Gamma_{111}^{D E D}+\Gamma_{000}^{D E D}+\Gamma_{101}^{D E D}+3 \Gamma_{110}^{D E D}-\Gamma_{011}^{D E D} \\
& +\Gamma_{100}^{D E D}+3 \Gamma_{001}^{D E D}+3 \Gamma_{010}^{D E D}
\end{aligned}
$$

$$
\begin{aligned}
\Gamma_{p}^{D E D}= & -5 \Gamma_{111}^{D E D}+\Gamma_{000}^{D E D}+5 \Gamma_{101}^{D E D}-3 \Gamma_{110}^{D E D}+\Gamma_{011}^{D E D} \\
& -\Gamma_{100}^{D E D}-\Gamma_{001}^{D E D}+3 \Gamma_{010}^{D E D} .
\end{aligned}
$$

$$
\text { 3. } \Gamma_{\mathbf{n}(\mathbf{p})}^{\mathrm{EDD}}
$$

The $\mathcal{W}_{\tau_{\Lambda^{\prime}} \tau_{\Lambda} \tau_{N}}^{E D D}\left(q, q^{\prime}, t\right)$ function, where $q^{\prime}=k-q-h^{\prime}$ and $t=q$, is identical to the $\mathcal{S}_{\tau^{\prime} \tau_{N} \tau}^{d d e}\left(q, q^{\prime}, t\right)$ function in Eq. (A3) of Ref. [24]. Moreover,

$$
\begin{aligned}
\Theta & \left.k, q, q^{\prime}, t, h, h^{\prime}, k_{F}\right) \\
= & \theta\left(q_{0}\right) \theta\left(|\mathbf{k}-\mathbf{q}|-k_{F}\right) \theta\left(|\mathbf{q}-\mathbf{h}|-k_{F} \mid\right) \\
& \times \theta\left(k_{F}-|\mathbf{h}|\right) \theta\left(\left|\mathbf{q}+\mathbf{h}^{\prime}\right|-k_{F} \mid\right) \theta\left(k_{F}-\left|\mathbf{h}^{\prime}\right|\right), \\
\varepsilon_{2 \mathrm{p} 2 \mathrm{~h}}^{E D D}= & \varepsilon_{2 \mathrm{p} 2 \mathrm{~h}}^{D D D},
\end{aligned}
$$

and $n=1$. The isospin sums are given by

$$
\begin{aligned}
\Gamma_{n}^{E D D}= & -\Gamma_{111}^{E D D}+\Gamma_{000}^{E D D}-\Gamma_{101}^{E D D}+3 \Gamma_{110}^{E D D}+\Gamma_{011}^{E D D} \\
& +3 \Gamma_{100}^{E D D}+\Gamma_{001}^{E D D}+\Gamma_{010}^{E D D}, \\
\Gamma_{p}^{E D D}= & -5 \Gamma_{111}^{E D D}+\Gamma_{000}^{E D D}+\Gamma_{101}^{E D D}-3 \Gamma_{110}^{E D D}+5 \Gamma_{011}^{E D D} \\
& +3 \Gamma_{100}^{E D D}-\Gamma_{001}^{E D D}-\Gamma_{010}^{E D D} .
\end{aligned}
$$

\section{4. $\Gamma_{\mathbf{n}(\mathbf{p})}^{\mathrm{DEE}}$}

The $\mathcal{W}_{\tau_{\Lambda^{\prime}} \tau_{\Lambda} \tau_{N}}^{D E E}\left(q, q^{\prime}, t\right)$ function, where $q^{\prime}=k-h$ and $t=h-h^{\prime}-q$, is identical to the $\mathcal{S}_{\tau^{\prime} \tau_{N} \tau}^{\text {eed }}\left(q, q^{\prime}, t\right)$ function in Eq. (A7) of Ref. [24]. Moreover,

$$
\begin{aligned}
\Theta( & \left.k, q, q^{\prime}, t, h, h^{\prime}, k_{F}\right) \\
= & \theta\left(q_{0}\right) \theta\left(|\mathbf{k}-\mathbf{q}|-k_{F}\right) \theta\left(|\mathbf{q}-\mathbf{h}|-k_{F} \mid\right) \\
& \times \theta\left(k_{F}-|\mathbf{h}|\right) \theta\left(\left|\mathbf{q}+\mathbf{h}^{\prime}\right|-k_{F} \mid\right) \theta\left(k_{F}-\left|\mathbf{h}^{\prime}\right|\right), \\
\varepsilon_{2 \mathrm{p} 2 \mathrm{~h}}^{D E E}= & \varepsilon_{2 \mathrm{p} 2 \mathrm{~h}}^{D D},
\end{aligned}
$$

and $n=1$. The isospin sums are given by

$$
\begin{aligned}
\Gamma_{n}^{D E E}= & 5 \Gamma_{111}^{D E E}+\Gamma_{000}^{D E E}+\Gamma_{101}^{D E E}+\Gamma_{110}^{D E E}+5 \Gamma_{011}^{D E E} \\
& +\Gamma_{100}^{D E E}+\Gamma_{001}^{D E E}+\Gamma_{010}^{D E E} \\
\Gamma_{p}^{D E E}= & -2 \Gamma_{111}^{D E E}-4 \Gamma_{101}^{D E E}+4 \Gamma_{110}^{D E E}+2 \Gamma_{011}^{D E E}+2 \Gamma_{100}^{D E E} \\
& +2 \Gamma_{001}^{D E E}+2 \Gamma_{010}^{D E E} .
\end{aligned}
$$

\section{5. $\Gamma_{\mathbf{n}(\mathbf{p})}^{\mathrm{EDE}}$}

The $\mathcal{W}_{\tau_{\Lambda^{\prime}} \tau_{\Lambda} \tau_{N}}^{E D E}\left(q, q^{\prime}, t\right)$ function, where $q^{\prime}=k-q-h^{\prime}$ and $t=h^{\prime}-h+q$, is identical to the $\mathcal{S}_{\tau^{\prime} \tau_{N} \tau}^{\text {eed }}\left(q, q^{\prime}, t\right)$ function in Eq. (A7) of Ref. [24]. Moreover,

$$
\begin{aligned}
& \Theta\left(k, q, q^{\prime}, t, h, h^{\prime}, k_{F}\right) \\
&= \theta\left(q_{0}\right) \theta\left(|\mathbf{k}-\mathbf{q}|-k_{F}\right) \theta\left(|\mathbf{q}-\mathbf{h}|-k_{F} \mid\right) \\
& \times \theta\left(k_{F}-|\mathbf{h}|\right) \theta\left(\left|\mathbf{q}+\mathbf{h}^{\prime}\right|-k_{F} \mid\right) \theta\left(k_{F}-\left|\mathbf{h}^{\prime}\right|\right), \\
& \varepsilon_{2 \mathrm{p} 2 \mathrm{~h}}^{E D E}= \varepsilon_{2 \mathrm{p} 2 \mathrm{~h}}^{\mathrm{DD}},
\end{aligned}
$$

and $n=1$. The isospin sums are given by

$$
\begin{aligned}
\Gamma_{n}^{E D E}= & -\Gamma_{111}^{E D E}+\Gamma_{000}^{E D E}+3 \Gamma_{101}^{E D E}+\Gamma_{110}^{E D E}-\Gamma_{011}^{E D E} \\
& +\Gamma_{100}^{E D E}+3 \Gamma_{001}^{E D E}+\Gamma_{010}^{E D E}
\end{aligned}
$$




$$
\begin{aligned}
\Gamma_{p}^{E D E}= & 4 \Gamma_{111}^{E D E}+6 \Gamma_{101}^{E D E}-4 \Gamma_{110}^{E D E}-2 \Gamma_{011}^{E D E} \\
& +2 \Gamma_{100}^{E D E}+2 \Gamma_{010}^{E D E}
\end{aligned}
$$

$$
\text { 6. } \Gamma_{\mathbf{n}(\mathbf{p})}^{\mathrm{EED}}
$$

The $\mathcal{W}_{\tau_{\Lambda^{\prime}} \tau_{\Lambda} \tau_{N}}^{\mathrm{EED}}\left(q, q^{\prime}, t\right)$ function, where $q^{\prime}=k-h$ and $t=k-q-h^{\prime}$, is identical to the $\mathcal{S}_{\tau^{\prime} \tau_{N} \tau}^{e d e}\left(q, q^{\prime}, t\right)$ function in Eq. (A5) of Ref. [24]. Moreover,

$$
\begin{aligned}
\Theta( & \left.k, q, q^{\prime}, t, h, h^{\prime}, k_{F}\right) \\
= & \theta\left(q_{0}\right) \theta\left(|\mathbf{k}-\mathbf{q}|-k_{F}\right) \theta\left(\left|\mathbf{q}+\mathbf{h}+\mathbf{h}^{\prime}-\mathbf{k}\right|-k_{F} \mid\right) \\
& \times \theta\left(k_{F}-|\mathbf{h}|\right) \theta\left(\left|\mathbf{q}+\mathbf{h}^{\prime}\right|-k_{F} \mid\right) \theta\left(k_{F}-\left|\mathbf{h}^{\prime}\right|\right), \\
\varepsilon_{2 \mathrm{p} 2 \mathrm{~h}}^{\mathrm{EED}}= & k_{0}-\varepsilon_{\mathbf{h}}+\varepsilon_{\mathbf{q}+\mathbf{h}+\mathbf{h}^{\prime}-\mathbf{k}}-\varepsilon_{\mathbf{q}+\boldsymbol{h}^{\prime}}-V_{N},
\end{aligned}
$$

and $n=1$. The isospin sum are given by

$$
\begin{aligned}
\Gamma_{n}^{E E D}= & -\Gamma_{111}^{E E D}+\Gamma_{000}^{E E D}+\Gamma_{101}^{E E D}+3 \Gamma_{110}^{E E D}-\Gamma_{011}^{E E D} \\
& +\Gamma_{100}^{E E D}+\Gamma_{001}^{E E D}+3 \Gamma_{010}^{E E D}, \\
\Gamma_{p}^{E E D}= & 4 \Gamma_{111}^{E E D}-4 \Gamma_{101}^{E E D}+6 \Gamma_{110}^{E E D}-2 \Gamma_{011}^{E E D} \\
& +2 \Gamma_{100}^{E E D}+2 \Gamma_{001}^{E E D} .
\end{aligned}
$$

\section{7. $\Gamma_{\mathbf{n}(\mathbf{p})}^{\mathrm{EEE}}$}

The $\mathcal{W}_{\tau_{\Lambda^{\prime}} \tau_{\Lambda} \tau_{N}}^{E E E}\left(q, q^{\prime}, t\right)$ function, where $q^{\prime}=k-h$ and $t=h+q-k$, is identical to the $\mathcal{S}_{\tau^{\prime} \tau_{N} \tau}^{e e e}\left(q, q^{\prime}, t\right)$ function in Eq. (A9) of Ref. [24]. Moreover,

$$
\begin{aligned}
& \Theta\left(k, q, q^{\prime}, t, h, h^{\prime}, k_{F}\right) \\
&= \theta\left(q_{0}\right) \theta\left(|\mathbf{k}-\mathbf{q}|-k_{F}\right) \theta\left(\left|\mathbf{h}^{\prime}+\mathbf{q}\right|-k_{F} \mid\right) \theta\left(k_{F}-\left|\mathbf{h}^{\prime}\right|\right) \\
& \times \theta\left(k_{F}-\left|\mathbf{k}-\mathbf{h}+\mathbf{h}^{\prime}\right|\right) \theta\left(k_{F}-|\mathbf{h}|\right),
\end{aligned}
$$

$$
\varepsilon_{2 \mathrm{p} 2 \mathrm{~h}}^{E E E}=k_{0}-\varepsilon_{\mathbf{h}}+\varepsilon_{\mathbf{h}^{\prime}}-\varepsilon_{\mathbf{k}-\mathbf{h}+\mathbf{h}^{\prime}}-V_{N},
$$

and $n=0$. The isospin sums are given by

$$
\begin{aligned}
\Gamma_{n}^{E E E}= & 5 \Gamma_{111}^{E E E}+\Gamma_{000}^{E E E}+\Gamma_{101}^{E E E}+\Gamma_{110}^{E E E}+5 \Gamma_{011}^{E E E} \\
& +\Gamma_{100}^{E E E}+\Gamma_{001}^{E E E}+\Gamma_{010}^{E E E}, \\
\Gamma_{p}^{E E E}= & 7 \Gamma_{111}^{E E E}+\Gamma_{000}^{E E E}+5 \Gamma_{101}^{E E E}+5 \Gamma_{110}^{E E E}+\Gamma_{011}^{E E E} \\
& -\Gamma_{100}^{E E E}-\Gamma_{001}^{E E E}-\Gamma_{010}^{E E E} .
\end{aligned}
$$

\section{APPENDIX B}

The explicit expressions of the function $\mathcal{R}\left(q_{0}, \mathbf{q}\right)$ of Eq. (22) reads

$$
\begin{aligned}
\mathcal{R}\left(q_{0}, \mathbf{q}\right) & =\frac{\pi}{(2 \pi)^{3}} \frac{m}{q}\left\{\frac { m ^ { 2 } } { q ^ { 2 } } \left[2\left(q_{0}-\frac{q^{2}}{2 m}\right) \frac{q}{m} k_{F}\right.\right. \\
& \left.+\left(\left(q_{0}-\frac{q^{2}}{2 m}\right)^{2}-\frac{q^{2}}{m^{2}} k_{F}^{2}\right) \ln \left|\frac{2 m q_{0}-q^{2}-2 q k_{F}}{2 m q_{0}-q^{2}+2 q k_{F}}\right|\right] \\
& +\theta\left(2 k_{F}-q\right)\left[-\frac{m^{2}}{q^{2}}\left(2 q_{0} \frac{q}{m}\left(k_{F}-\frac{q}{2}\right)+\left(q_{0}^{2}-\frac{q^{2}}{m^{2}}\right.\right.\right. \\
& \left.\left.\times\left(k_{F}-\frac{q}{2}\right)^{2}\right) \ln \left|\frac{2 m q_{0}+q^{2}-2 q k_{F}}{2 m q_{0}-q^{2}+2 q k_{F}}\right|\right) \\
& \left.+q\left(\frac{q}{4}-k_{F}\right) \ln \left|\frac{2 m q_{0}-q^{2}+2 q k_{F}}{2 m q_{0}+q^{2}-2 q k_{F}}\right|\right) \\
& \left.\left.-q_{0} m \ln \left|\frac{q_{0}^{2} m^{2}-q^{2}\left(k_{F}-q / 2\right)^{2}}{m^{2} q_{0}^{2}}\right|\right]\right\},
\end{aligned}
$$

where $q=|\mathbf{q}|$ and $m$ is the nucleon mass.
[1] For a recent collection of reviews on nuclear physics with strangeness see the Special Issue on Recent Advances in Strangeness Nuclear Physics, edited by A. Gal and R. S. Hayano [Nucl. Phys. A 804, 1 (2008)].

[2] E. Oset and A. Ramos, Prog. Part. Nucl. Phys. 41, 191 (1998).

[3] W. M. Alberico and G. Garbarino, Phys. Rep. 369, 1 (2002); in Hadron Physics, edited by T. Bressani, A. Filippi, and U. Wiedner (IOS Press, Amsterdam, 2005), p. 125. For an update of these reviews see Ref. [4].

[4] C. Chumillas, G. Garbarino, A. Parreño, and A. Ramos, Nucl. Phys. A 804, 162 (2008).

[5] A. Parreño, Lect. Notes Phys. 724, 141 (2007).

[6] H. Outa, in Hadron Physics, edited by T. Bressani, A. Filippi, and U. Wiedner (IOS Press, Amsterdam, 2005), p. 219.

[7] J. Nieves and E. Oset, Phys. Rev. C 47, 1478 (1993); T. Motoba and K. Itonaga, Prog. Theor. Phys. Suppl. 117, 477 (1994); T. Motoba, K. Itonaga, and H. Bando, Nucl. Phys. A 489, 683 (1988).

[8] A. Gal, Nucl. Phys. A 828, 72 (2009).

[9] G. Garbarino, A. Parreño, and A. Ramos, Phys. Rev. Lett. 91, 112501 (2003).

[10] G. Garbarino, A. Parreño, and A. Ramos, Phys. Rev. C 69, 054603 (2004).
[11] A. Ramos, M. J. Vicente-Vacas, and E. Oset, Phys. Rev. C 55, 735 (1997); 66, 039903(E) (2002).

[12] W. M. Alberico, A. De Pace, M. Ericson, and A. Molinari, Phys. Lett. B 256, 134 (1991).

[13] A. Ramos, E. Oset, and L. L. Salcedo, Phys. Rev. C 50, 2314 (1994)

[14] W. M. Alberico, A. De Pace, G. Garbarino, and A. Ramos, Phys. Rev. C 61, 044314 (2000).

[15] W. M. Alberico, A. De Pace, G. Garbarino, and R. Cenni, Nucl. Phys. A 668, 113 (2000).

[16] E. Bauer and F. Krmpotić, Nucl. Phys. A 739, 109 (2004).

[17] E. Bauer, Nucl. Phys. A 818, 174 (2009).

[18] E. Bauer and G. Garbarino, Nucl. Phys. A 828, 29 (2009).

[19] E. Bauer, G. Garbarino, A. Parreño, and A. Ramos, Nucl. Phys. A 836, 199 (2010).

[20] E. Bauer and F. Krmpotić, Nucl. Phys. A 717, 217 (2003).

[21] B. H. Kang et al., Phys. Rev. Lett. 96, 062301 (2006).

[22] M. J. Kim et al., Phys. Lett. B 641, 28 (2006).

[23] E. Bauer, Nucl. Phys. A 781, 424 (2007).

[24] E. Bauer, Nucl. Phys. A 796, 11 (2007).

[25] E. Bauer and G. Garbarino, Nucl. Phys. A 835, 430 (2010).

[26] E. Oset and L. L. Salcedo, Nucl. Phys. A 443, 704 (1985). 
[27] A. Parreño, A. Ramos, and C. Bennhold, Phys. Rev. C 56, 339 (1997); A. Parreño and A. Ramos, ibid. 65, 015204 (2001).

[28] V. G. J. Stoks and Th. A. Rijken, Phys. Rev. C 59, 3009 (1999); Th. A. Rijken, V. G. J. Stoks, and Y. Yamamoto, Phys. Rev. C 59, 21 (1999).

[29] R. Machleidt, K. Holinde, and Ch. Elster, Phys. Rep. 149, 1 (1987).

[30] M. B. Barbaro, A. De Pace, T. W. Donnelly, and A. Molinari, Nucl. Phys. A 596, 553 (1996).

[31] M. Kim et al., Phys. Rev. Lett. 103, 182502 (2009).

[32] M. Agnello et al., Phys. Lett. B 685, 247 (2010).

[33] W. Cassing et al., Eur. Phys. J. A 16, 549 (2003).

[34] Y. Sato et al., Phys. Rev. C 71, 025203 (2005).
[35] H. Outa, contributed talk at the PANIC08 conference, Eilat (Israel), 9-14 November 2008; H. Outa et al., Nucl. Phys. A 754, 157c (2005).

[36] M. Agnello et al., Phys. Lett. B 622, 35 (2005); Nucl. Phys. A 804, 151 (2008).

[37] T. Nagae, Nucl. Phys. A 754, $443 \mathrm{c}$ (2005); 805, 486c (2008).

[38] S. Ajimura et al., Exclusive Study on the $\Lambda N$ Weak Interaction in $A=4 \Lambda$ Hypernuclei, Letter of intent for an experiment (E22) at J-PARC (2007); H. Bhang et al., Coincidence Measurement of the Weak Decay of ${ }_{\Lambda}^{12} \mathrm{C}$ and the Three-Body Weak Interaction Process, Letter of intent for an experiment (E18) at J-PARC (2006).

[39] T. Fukuda et al., Nucl. Phys. A 790, 161c (2007). 